\title{
NOTES ON THE DESIGN OF 4-TERMINAL RESISTANCE STANDARDS FOR ALTERNATING CURRENTS
}

\author{
By Francis B. Silsbee
}

\section{ABSTRACT}

The design of a resistance standard for use with alternating current involves not only the consideration of the value, definiteness, and permanence of the resistance and the adequate cooling of the metal parts, which are encountered in direct-current standards as well, but also the consideration of the inductance and of the possible change in the resistance of the standard with the frequency of the current flowing in it. These notes give the theoretical basis for the computation of the inductance of resistance standards which consist of a system of straight conductors long in comparison to their diameters. Most standards used in the measurement of large currents are of this type. Formulas are given for the skin effect in various combinations of flat strips and coaxial tubes. Methods of attaching and locating the potential leads so as to minimize the possibility of error from stray magnetic fields and yet permit of convenient adjustment of the resistance are discussed. As examples of the principles here set forth, detailed descriptions are given of two groups of resistance standards which have been constructed at the Bureau of Standards for testing current transformers. These standards range in resistance from 0.05 to $0.0002 \mathrm{ohm}$ and in current capacity from 10 to 2,500 amperes.

\section{CONTENTS}

I. Introduction

II. Theoretical relations

III. End effects

IV. Attachment of potential leads

V. Skin effect... 85

VI. Air-cooled resistance standards

VII. Oil-cooled resistance standards 100

VIII. Conclusions

IX. Notation .

X. Appendix A. Formulas for geometric mean distances..... 104

\section{INTRODUCTION}

The constantly increasing use of electrical energy in large amounts has given more and more importance to the problem of accurately measuring alternating currents of large value. Such measurements usually involve the use of a current transformer, the secondary winding of which serves to supply to the measuring instruments proper a current of convenient amount, which bears a definite relation in magnitude and phase to the much larger current flowing in the primary winding. The exact determination of the relation of secondary to primary currents must be determined by suitable measurements, which in most cases directly or indirectly require the use of resistance standards or "shunts" which are capable of carrying the primary current and which have definite and accurately known resistance and inductance.

The Bureau of Standards has given considerable attention to the design of such resistance standards, and Scientific Paper ${ }^{2}$ No. 281,

\footnotetext{
1 In this paper the term "shunt" will be used in place of the inconvenient though, perhaps, more nearly correct phrase "4-terminal resistance standard."

2 B. S. Bull. 13, p. 375 .
} 
published in 1916, describes the results of our earlier work on this subject. The purpose of the present paper is to supplement the earlier one (a) by presenting the basis for the theoretical computation of inductance from a somewhat different point of view, (b) by reporting the results of further experimental work bearing on some possible sources of error in such measurements, and (c) by describing a series of shunts of two different types which have been constructed at the bureau in recent years.

The computation of the inductance of systems of conductors having various geometrical configurations is useful in a number of applications. One of these is to determine with high precision the inductance of shunts which are to be used primarily as standards of inductance with which other shunts are to be compared. It often happens, however, that considerations of convenience or adjustability require that a working standard of resistance have a shape which departs considerably from any of those for which it is feasible to carry through an exact mathematical calculation of inductance. Such shunts must be regarded as secondary standards and for precise work must be compared experimentally with primary inductance standards. Nevertheless, it is frequently desirable to be able to estimate roughly the inductance to be expected with such a type of construction, and the procedure given below may in many cases be applied to give such results with sufficient accuracy for the purpose at hand.

In the following pages will be found a derivation of the geometric mean distance formula on which the inductance computations are based with instructions for applying this to conductors of various types. A discussion of the extent to which conditions in an actual standard may depart from the ideal as the result of skin effect, end effect, attachment of potential leads, etc., is then given. It is then shown how these principles can be applied to the design of a new type of air-cooled flat-strip shunt; and a brief description is given of some tubular oil-cooled shunts recently completed.

\section{THEORETICAL BASIS FOR CALCULATION OF INDUC- TANCE}

A general definition of the inductance of a 4-terminal resistor is obtained by considering that a sinusoidal alternating current $I$ flows through the resistor from terminal 1 to terminal 2 , and that an alternating electromotive force $E$ then appears ${ }^{3}$ between terminals 3 and 4. If the vector ratio of $E$ to $I$ is given by

$$
\frac{E}{I}=Z=R^{\prime}+j \omega L
$$

where $\omega=2 \pi \times$ frequency; then $L$ is the inductance of the resistor, and $R^{\prime}$ is its alternating-current resistance. The conditions which must be satisfied to make $L$ a definite quantity are discussed at length elsewhere $;^{4}$ but the principal requirements are that the potential terminals 3 and 4 shall be located at a considerable distance from the current terminals 1 and 2 , and that the two terminals of each pair shall be as close together as possible. 
In considering problems involving the characteristics of 4-terminal resistors, two very general principles are often of great value. The first of these is the "reciprocal theorem" which states that if current is passed through terminals 3 and 4 and the resulting electromotive force is observed at terminals 1 and 2 the resistance and inductance will have the same numerical values as if the converse choice of terminals had been made and the electromotive force had been observed at 3 and 4 when current was passing through 1 and 2. This theorem is exceedingly convenient in discussing the inductance of complicated groups of conductors, since that pair of terminals can be considered as current terminals which yields the simplest set of streamlines and equipotential surfaces.

The second useful conception is that of regarding the two possible current paths (that through 1 and 2 and that through 3 and 4 ) as separate and distinct circuits, which can be called the "current circuit" and the "potential circuit," respectively. The self-inductance of the complete resistor then becomes the same as the mutual inductance between the two circuits so visualized. The fact that throughout part of their volumes the two circuits coincide in space does not affect the argument. When regarded as a mutual inductance, it is easier to see that the total inductance is the algebraic sum of the mutual actions of the several parts of the one circuit upon those of the other. The effect of an external stray field on the resistor can be considered as a mutual inductance between the potential circuit and the circuit which produces the external field.

Most shunts intended for use with alternating currents approximate more or less closely to an ideal configuration of conductors, for which the mathematical process of summing up the mutually inductive effects of each part of the current circuit on each part of the potential circuit can be carried through with comparative ease. The accuracy with which the actual inductance of the shunt agrees with the inductance computed for the ideal configuration depends, of course, on the fidelity with which the actual construction approaches the ideal configuration. The configuration approximated in the design of many alternating current shunts is that of a group of infinitely long parallel straight conductors of uniform cross section and of homogeneous, isotropic material. In any conductor of such a group, the streamlines of current are necessarily straight and parallel to the bounding surfaces of the conductor, and the potential gradient has a constant value at all points of any one conductor, both along its length and over its cross section. If the frequency is sufficiently low, the current density will also be uniform over the cross section. The inductance formulas given in this (and in most other papers) are based on this assumption of uniform current distribution.

Numerical computations of the inductance of systems of straight parallel conductors can most readily be based upon the following derivation ${ }^{6}$ of the mutual inductance between two circuits of this type.

Figure 1 shows the cross section of four such conductors. Consider that conductors 1 and 2 of area $S_{1}$ and $S_{2}$ form the outgoing

5 Kirchhoff, Pogg. Ann., 72, 1847.

5 The derivation here given closely follows the presentation in Orlich's "Kapazität und Induktivität," p. 68, F. Vieweg u. Sohn, Leipzig; 1909. 
and return conductors of a single circuit which carries a current $I_{p}$. Similarly, consider that conductors 3 and 4 of areas $S_{3}$ and $S_{4}$ constitute a second circuit which carries a current $I_{s}$. If $i_{1}, i_{2}, i_{3}$, and $i_{4}$ are the current densities in conductors $1,2,3$, and 4, respectively, counted positive when in the same direction in all conductors, and

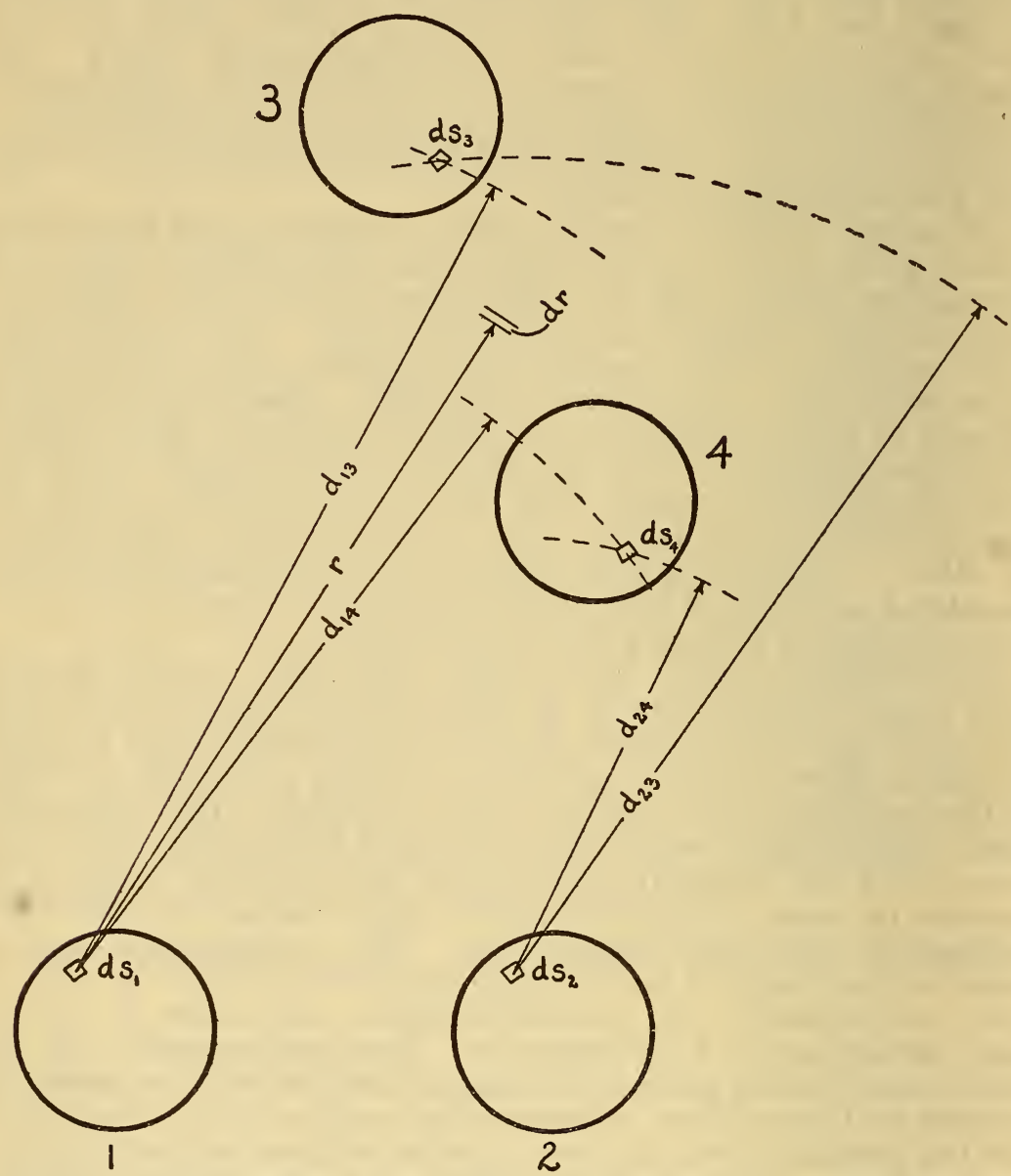

FIGURE 1.-Cross section of four long straight conductors illustrating calculation of mutual inductance

the currents are uniformly distributed over the cross sections of the conductors

and

$$
\begin{aligned}
& i_{1} S_{1}=-i_{2} S_{2}=I_{p} \\
& i_{3} S_{3}=-i_{4} S_{4}=I_{s}
\end{aligned}
$$

Consider each conductor divided into a number of infinitesimal filaments, their cross sections $d S_{1}, d S_{2}, d S_{3}$, and $d S_{4}$ being so chosen that the current $i_{2} d S_{2}$ in a filament of conductor 2 may be considered as the return of the current element $i_{1} d S_{1}$ in conductor 1 , and similarly for conductors 3 and 4 . 
Now, the flux for a length $l$ of circuit, due to the current in filament $d S_{1}$ linking the circuit formed by $d S_{3}$ and $d S_{4}$ is

$$
\imath \int_{d_{13}}^{d_{14}} \frac{2 i_{1} d S_{1}}{r} d r=2 i_{1} l d S_{1}\left(\log _{e} d_{14}-\log _{e} d_{13}\right)
$$

where $r$ is a radial distance from $d S_{1}$.

$d_{13}$ and $d_{14}$ are the distances from $d S_{1}$ to $d S_{3}$ and $d S_{4}$, respectively.

The corresponding number of flux linkages due to the current in $d S_{2}$ is similarly

$$
2 i_{2} l d S_{2}\left(\log _{e} d_{24}-\log _{e} d_{23}\right)
$$

The mutual magnetic energy corresponding to these linkages is obtained by multiplying the total number of linkages by the element of current $i_{3} d S_{3}=-i_{4} d S_{4}$ which is linked with it. This magnetic energy is therefore

$$
\begin{gathered}
d W=2 l\left[i_{1} d S_{1}\left(-i_{4} d S_{4} \log _{e} d_{14}-i_{3} d S_{3} \log _{e} d_{13}\right)+i_{2} d S_{2}\left(-i_{4} d S_{4} \log _{e} d_{24}\right.\right. \\
\left.\left.-i_{3} d S_{3} \log _{e} d_{23}\right)\right]
\end{gathered}
$$

On substituting for the various current densities their value in terms of the total currents from equation (2) this becomes

$$
\begin{array}{r}
d W=2 l I_{p} I_{s}\left[\frac{1}{S_{1} S_{4}} \log _{e} d_{14} d S_{1} d S_{4}-\frac{1}{S_{1} S_{3}} \log _{e} d_{13} d S_{1} d S_{3}\right. \\
\left.-\frac{1}{S_{2} S_{4}} \log _{e} d_{24} d S_{2} d S_{4}+\frac{1}{S_{2} S_{3}} \log _{e} d_{23} d S_{2} d S_{3}\right]
\end{array}
$$

The total mutual magnetic energy is obtained by adding all these elementary contributions-that is, by integrating over the cross sections of the conductors. This total energy can also be expressed in terms of the mutual inductance $M$ of the two circuits as

$$
W=M I_{p} I_{s}
$$

Combining equations (5) and (6) and indicating the integrations gives

$$
\begin{aligned}
M=2 d & \frac{1}{S_{1} S_{4}} \int_{S_{1}} \int_{S_{4}} \log _{e} d_{14} d S_{1} d S_{4}-\frac{1}{S_{1} S_{3}} \int_{S_{1}} \int_{S_{3}} \log _{e} d_{13} d S_{1} d S_{3} \\
& -\frac{1}{S_{2} S_{4}} \int_{S_{2}} \int_{S_{4}} \log _{e} d_{24} d S_{2} d S_{4}+\frac{1}{S_{2} S_{3}} \int_{S_{2}} \int_{S_{3}} \log _{e} d_{23} d S_{2} d S_{3}
\end{aligned}
$$

Now, the definition of the geometric mean distance $D_{x y}$ between two areas $S_{x}$ and $S_{y}$ is

$$
\log _{e} D_{x y}=\frac{1}{S_{x} S_{y}} \int_{S_{1}} \int_{S_{3}} \log _{e} d_{x y} d S_{x} d S_{y}
$$

and also the mutual inductance is defined as the ratio of secondary flux linkages to primary current, so that

$$
M=\frac{\phi}{I}=2 l\left[\log _{e} D_{14}-\log _{e} D_{13}-\log _{e} D_{24}+\log _{e} D_{23}\right]
$$

or

$$
=2 l \log _{e} \frac{D_{14} D_{23}}{D_{13} D_{24}}
$$


In the foregoing linkages have been counted as positive when due to the current in 1 and when threading circuit 3 and 4 in the same direction as if due to a current in 3 which is in the same direction as the current in 1 . The sign of $M$ as given by equation (9) is therefore such that if conductors 2 and 3 were joined and a current sent from 1 to 4 through the series circuit thus formed the total selfinductance would be given by

$$
L=L_{12}+L_{34}+2 M_{12,34}
$$

If we wish to pass from the mutual inductance of two separate circuits to the self-inductance of a 4-terminal resistor, one or more of the conductors of the potential circuit must be considered as coinciding with the corresponding conductor of the current circuit: Thus, the self-inductance of the circuit formed by the two conductors 1 and 2 only, is obtained if we imagine conductor 3 to coincide with 1 and 4 with 2. Equation (9) then becomes

$$
L=2 l \log _{e} \frac{D^{2}{ }^{2}}{D_{11} D_{12}}
$$

where the symbol $D_{11}$ indicates the geometric mean distance of the cross section of conductor 1 from itself.

In case conductors 1 and 2 are equal, equation (11) reduces to the usual formula.

$$
L=4 l \log _{e} \frac{D_{12}}{D_{11}}
$$

An actual resistance standard will unavoidably depart from the ideal configuration in a number of respects. The departure from the condition of infinite length is discussed below in the section on "end effects," and the departure from uniform current distribution in the section on "skin effect." Effects arising from local inhomogeneities in the conductivity of the metal are, of course, not amenable to mathematical treatment. In certain cases, however, such as concentric tubular shunts, the existence of any resulting irregularity in current distribution can be detected by sending an alternating current through the shunt and exploring the magnetic field with a search coil connected to a vibration galvanometer. Any departure from straightness is usually entirely negligible. In fact, the formulas deduced on the basis of the foregoing assumptions of long straight conductors may sometimes be applied with fairly good approximation to cases when the entire system of conductors is curved, provided only that the radius of curvature is large compared with the transverse dimensions of the conductors.

The proper sign for inductance can be obtained by applying the following procedure to a sketch of the system. Starting at one current terminal proceed along the current circuit, indicating by an arrow on each conductor an assumed direction of continuous current flow. Indicate by similar arrows on each conductor of the potential circuit such a direction that on the common conductors the arrows of the two sets have the same direction. Assign a number to each conductor in such a way that all conductors marked with arrows in one direction have odd numbers and those marked oppositely have 
even numbers. Group the conductors of each circuit into pairs so that the two members of each pair are marked with arrows pointing in opposite directions, and so that no conductor is assigned to more than one pair in the same current or potential circuit. If either or both circuits have an odd number of conductors, either $(a)$ the corresponding terminals are separated by the length of the resistor and its inductance is indefinite until the location of the return conductor is specified (for example, an ordinary resistance standard for direct current) or $(b)$ the terminals are close together as a result of the entire system being curved to a radius large compared with the transverse dimensions of the conductors (for example, the L. and N. adjustable secondary resistor). In this latter case the circuits having the odd number of conductors should be considered as having an additional conductor located at a great distance from the others, but serving to complete it. The geometric mean distances of this imaginary conductor from the members of each pair of conductors in the other circuit will always appear in equation (9) in such a way as to cancel each other.

For each pair of conductors in the current circuit combined with each pair of conductors in the voltage circuit compute a contribution to the total inductance by equation (9). The algebraic sum of these contributions is then the desired inductance. When substituting in equation (9) distances having like subscripts (that is, both even or both odd) must be in the denominator and those having unlike subscripts in the numerator.

The conductor cross sections used in resistance standards are almost invariably either rectangles or circular annuli or special cases of these, such as lines (for thin strips) or circles (round wires). The derivation of the formulas for the geometric mean distance between such shapes from the definition of G. M. D. is not difficult but is rather: laborious in the case of the rectangles. In the case of the circular shapes the evaluation of the definite integral ${ }^{7}$ which represents the geometric mean distance is given by the formula.

$$
\left.\int_{0}^{\pi} \log _{e}(k \pm t \cos y) d y=\pi \log _{e}\left(\frac{k+\sqrt{k^{2}-t^{2}}}{2}\right) ; k \geqq t\right)
$$

Bureau of Standards Scientific Paper ${ }^{8}$ No. 169 on pages 168 to 170 gives all the exact expressions for circular and annular G. M. D's.

In the case of thin-walled tubes the formulas can be much simplified. ${ }^{9}$ Scientific Papers No. 281, page 400, and No. 320, page 565, also give the simplified expressions for the inductance of shunts using several possible combinations of tubes.

The most general formula for the G. M. D. of rectangles placed obliquely but with their sides parallel, is given by Gray, ${ }^{10}$ but with certain misprints in the signs. These errors are corrected and the complete formulas for symmetrically located rectangles are given in Scientific Paper ${ }^{11}$ No. 47.

As special cases of these formulas or by direct integration other formulas can be obtained for more special cases, such as lines and points. The formulas for a number of such special cases are given below in Appendix A.

\footnotetext{
7 B. O. Peirce, A Short Table of Integrals, No. 523, p. 65.

8 B. S. Bull., 8; 1911

B. S. Bull., 14; 1918; B. S. Sci. Paper No. 320.

10 Absolute Measurements, 2 , pt. 1 , pp. 288-306.

11 B. S. Bull., 3, p. 6; 1907.
}

$84789^{\circ}-29-6$ 
As an example of the procedure outlined above we may consider a shunt of the type ${ }^{12}$ shown in Figure 2. Here the circuit between the current terminals $C_{1}$ and $C_{2}$ consists of the bars $A D$ and $D G$ of resistance material, joined at $D$. The potential circuit between $P_{1}$ and $P_{2}$ is connected to the current circuit at $B$ and $F$, and over the length $l_{1}$ the leads $B C$ and $F E$ are held parallel to the bars opposite their center line. The various dimensions are indicated on the cross section $X X^{\prime}$. From $E$ and $C$ the leads are brought off at right angles to the axis and are kept close together from there to the potential terminals.

The total inductance is, of course, the same as the mutual inductance between the total potential and current circuits, and this obviously consists of two parts contributed by the sections of length $l_{1}$ and $l_{2}$, respectively. Indicating directions and numbering the conductors in accordance with the rules given above shows that for the section
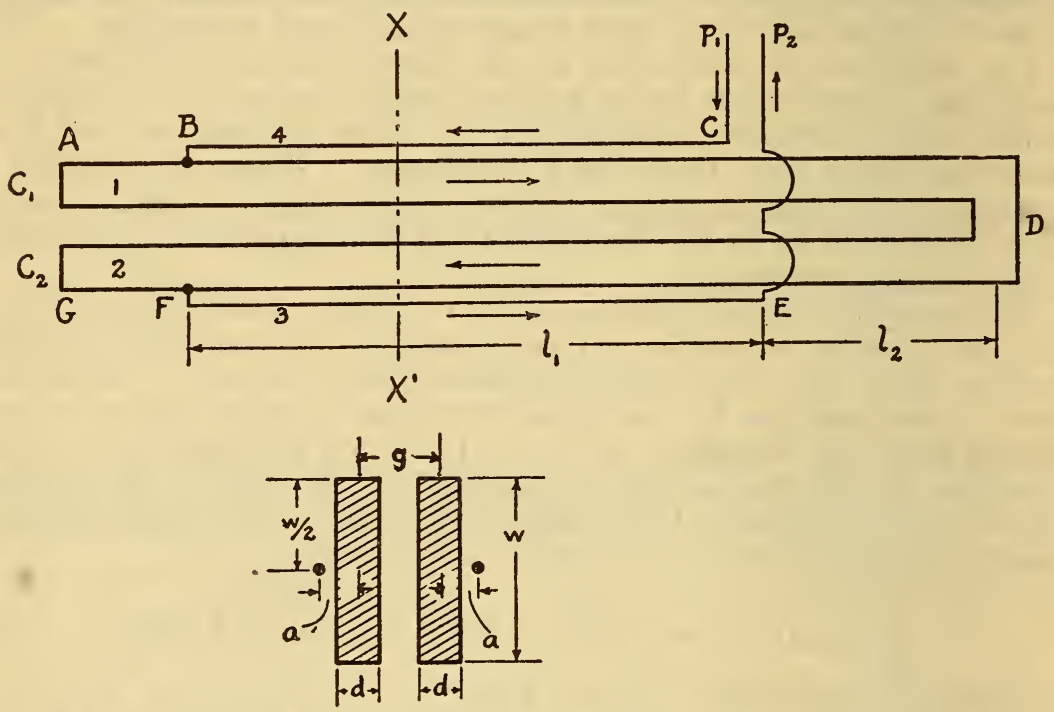

Section on $X X^{\prime}$

FIGURE 2.-Flat strip shunt with compensating potential leads

of length $l_{1}$ there is the one pair of current conductors 1 and 2 , as well as the two pairs of potential conductors 1 and 2 and 3 and 4 ; while for the section of length $l_{2}$ there is the one pair of current conductors 1 and 2, but only one pair of potential conductors 1 and 2 . We therefore have for the total inductance by equation (9)

$$
L=2 l_{1}\left[\log _{e} \frac{D_{12} \cdot D_{21}}{D_{11} \cdot D_{22}}+\log _{e} \frac{D_{14} \cdot D_{23}}{D_{13} \cdot D_{24}}\right]+2 l_{2}\left[\log _{e} \frac{D_{12} \cdot D_{21}}{D_{11} \cdot D_{22}}\right]
$$

Now, $D_{12}=D_{21}$ and also if the bars are of the same size and the potential leads are symmetrically placed $D_{11}=D_{22}, D_{14}=D_{23}, D_{13}=D_{24}$, hence,

$$
L=4 l_{1}\left[\log \frac{D_{12}}{D_{11}}+\log \frac{D_{14}}{D_{13}}\right]+4 l_{2} \log \frac{D_{12}}{D_{11}}
$$

12 This type of construction is used in certain apparatus for current-transformer testing made by the Leeds \& Northrup Co. 
Inserting the values of the various geometric mean distances as given in the appendix yields, in case the thickness and spacings are small compared to the width of the bars,

$$
L=4 l_{1}\left[\frac{\pi\left(g-\frac{1}{3} d\right)}{w}-\frac{\pi(g)}{w}\right]+4 l_{2}\left[\frac{\pi\left(g-\frac{1}{3} d\right)}{w}\right]
$$

or

$$
L=\frac{4 \pi}{w}\left[-\frac{1}{3} d l_{1}+\left(g-\frac{1}{3} d\right) l_{2}\right]
$$

This example brings out the fact that a fairly simple arrangement of the potential leads may introduce a compensating effect on the inductance of a shunt which may be of considerable utility. If the construction used in the section of length $l_{2}$ were used for the entire shunt, it would show a positive inductance of $+\frac{4 \pi}{w}\left(g-\frac{1}{3} d\right) l$ millimicrohenries. If, on the other hand, the construction used in the section of length $l_{1}$ was extended over the entire length, the inductance would be negative and have the value $-\frac{4 \pi d l}{3 w}$. Hence, by choosing the lengths in the ratio given by

$$
\frac{l_{1}}{l_{2}}=\frac{g-\frac{1}{3} d}{\frac{1}{3} d}
$$

a shunt can be constructed having zero effective inductance.

\section{END EFFECTS}

The resistors actually used in the measurement of heavy currents are far from being infinite in length, and it might be thought that the formulas discussed in the foregoing paragraphs would have little applicability. That this is not the case, can be seen from a study of Figure 3. Here $A B C D E F G H$ is the current circuit of a shunt of finite length, the potential circuit being $J K D C L M$. Let us superpose on this system the additional conductors shown in the dotted lines, which coincide with the actual conductors along $E F$ and $B G$, and which extend to an indefinitely great distance at $N P$ and $R S$. When currents are flowing in these additional conductors, as shown by the dotted arrows, the 4-terminal inductance of the shunt will be exactly that corresponding to a length $l$ of a system of infinitely long straight conductors and may be designated by $L_{\infty}$. If the inductance of the actual 4-terminal resistor is $L_{l}$, then the difference $L_{l}-L_{\infty}$ may be called the "end effect."

It is evident that this difference is the result of and is equal to the mutual inductance between the potential circuit $J K D C L M$ and the superposed loops $N E F P$ and $R B G S$. In most cases the transverse dimensions $a$ and $g$ are small compared with the working length $l$ of the shunt and this mutual inductance is very small. This is particularly true if the current circuit is made longer than the distance between potential taps as shown at $k$ and $m$. Measurements on shunts made of flat sheet material have shown end effects 
less than 10 per cent of the self-inductance of the shunt in cases where both the width and the spacing of the strips were as great as 80 per cent of the length of the shunt. It is therefore evident that the formulas derived on the basis of infinitely long conductors may be satisfactory guides even in cases of short, thick shunts.

The two forms of shunt most frequently chosen for use as computable standards of inductance are either a pair of flat strips close together or a pair of concentric tubes. Experiments ${ }^{13}$ on shunts of

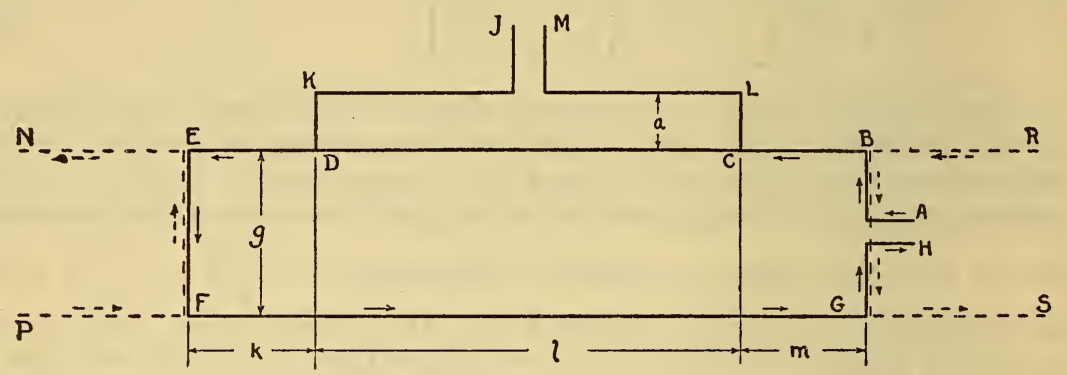

FIGURE 3.-Illustration of end-effects in four-terminal resistance standard

the former type have indicated that the end effect is quite negligible when the strips were close together and of reasonable length.

The end effect in shunts in which the current flows longitudinally in a pair of coaxial tubes of circular section joined at the ends by annular conductors is rigorously zero, provided that the magnetic field is not affected by the current flow in the current terminal blocks, and that the current is distributed uniformly around the axis. It will be seen that such a shunt is equivalent to the toroid formed by rotating a rectangle about an external axis which is parallel to the long sides of the rectangle.

It has been shown by Maxwell and others ${ }^{14}$ that such a toroidal circuit produces no magnetic field whatever at external points. Furthermore, the end extensions required to make the transition from the actual shunt to the ideal pair of infinitely long conductors also constitute similar toroids, and hence can produce no magnetic field and no electromotive force in the potential circuit.

\section{ATTACHMENT OF POTENTIAL LEADS}

In the case of shunts which are not intended to serve as computable standards of inductance, it is often convenient to attach the potential leads to small ears partially cut from the sheet manganin by a $U$-shaped slot as indicated in Figure 4 at $A$ and $B$. This form of construction has long ${ }^{15}$ been used in resistance standards and makes it possible either to increase or decrease the effective 4-terminal resistance of the standard over a moderate range by filing at the ends of the $U$ at $A$ or at $B$, respectively.

It will be seen, however, that this construction materially disturbs the streamlines of current over an area somewhat larger than the

13 B. S. Bull., 13, p. 396, 1916; B. S. Sci. Paper No. 281.

14 Maxwell, Electricity and Magnetism, 3d ed.. 2, p. 313, sec. 681; Webster, Electricity and Magnetism. p. $451 ; 1897$.

${ }_{15}$ W. Clark Fisher, The Potentiometer and Its Adjuncts, p. 72; 1902. 
entire ear, and thus in effect superposes on the normal magnetic field of the shunt a small local bundle of magnetic flux. This flux is linked with the potential circuit at one end of the shunt ( $A$, fig. 4$)$ and will, therefore, contribute a certain amount to the effective inductance of the standard. Approximate calculation and some measurements on an experimental shunt indicate that this effect, though small, may introduce errors amounting to more than 1 millimicrohenry. Consequently, this form of attachment should be avoided in shunts which are to be used as computable inductance standards.

An alternative arrangement which permits of adjustment of resistance in either direction over a sufficient range without producing an appreciable effect on the inductance is shown by the potential connections on the lower side of the tubular shunt in Figure 4. Lead $E L$ is soldered to the shunt at $E$. Leads $F$ and $G$ are similarly attached at such a distance from $E$ that the resistance between $E$ and $G$ is greater and that between $E$ and $F$ is less than the nominal resistance of the shunt. The leads from $F$ and $G$ are joined by a resistor $H K$, and the binding post $M$ is connected to an intermediate tap point $J$. Adjustment of the 4-terminal resistance is then obtained by unsolder-

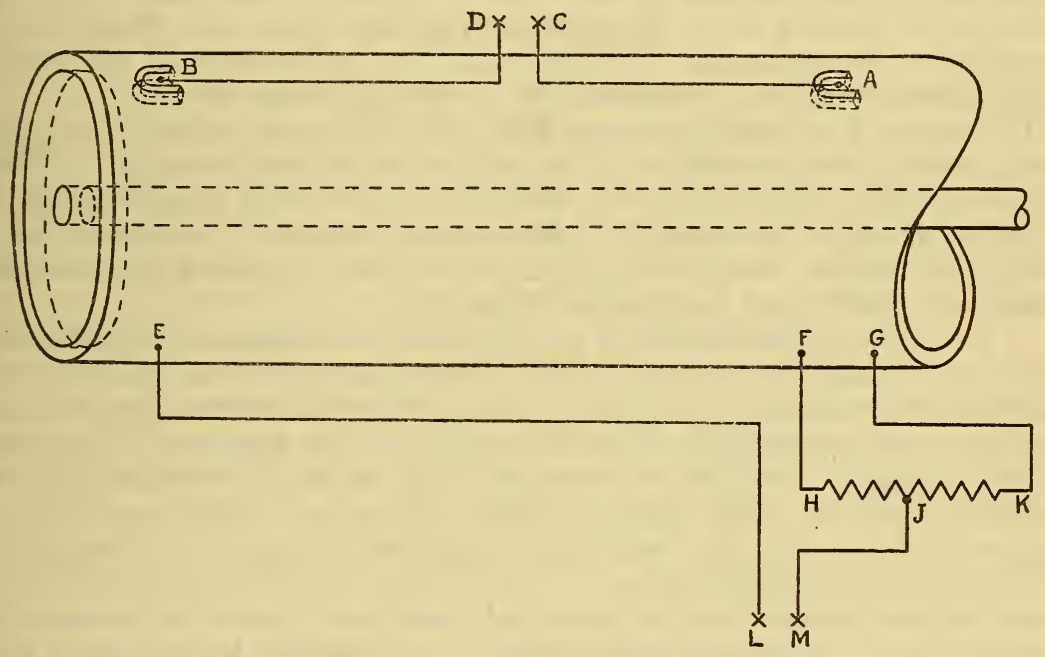

Figure 4.-Alternative methods of connecting potential leads so as to admit of resistance adjustment

ing tap $J$ and moving it to the desired position without tampering with the main current-carrying portion of the shunt. Of course, a certain current flows through the leads and the resistor $H K$, but this is very small, since $H K$ may be chosen to have a resistance several thousand times that of the part of the main conductor lying between $F$ and $G$. The possible range of adjustment is, of course, approximately $\frac{F G}{E G}$, and it can be shown that the effect of this potential circuit on the time constant of the shunt as a whole is always less than the product of this range of adjustment and the time constant of the circuit $F H K G$. Since this latter circuit is of fairly high resistance, it is easy to make the product of these two small factors negligibly small. 
The location of the potential leads in their course from the point where they are attached to the current circuit to the potential binding posts is usually fixed by the requirement that the 4-terminal inductance be as definite and as small as possible. In certain cases where the current-carrying parts are symmetrical there is still a certain latitude in the placing of the potential leads. This is especially true in the case the current circuit is formed of coaxial cylinders, for such a circuit produces no magnetic field at points outside or inside of both cylinders. The inductance is therefore the same for any location of the potential leads, provided they do not pass through the region between the tubes. However, if only a single pair of leads is attached to such a shunt, and if, for instance, only the outer tube is used as a resistor, the complete potential circuit may constitute a loop of considerable area, and hence be very susceptible to stray magnetic fields. If a second pair of potential leads is attached to the outer tube at points diametrically opposite to the points of attachment of the first pair, and if the two leads of each pair are tied down close to the elements of the cylinder joining their points of attachment, then the emf induced in one potential circuit by a uniform stray field is equal and opposite to that induced in the other. Hence, if the leads (of equal resistance) coming from the same end of the shunt are connected in parallel at the potential binding posts the inductance of the shunt thus formed will not be affected by a uniform stray field.

Of course, a nonuniform stray field will still cause an error, and the ideal arrangement would be to use a tubular potential sheath. ${ }^{16}$ This, however, with most forms of construction has the disadvantage of interfering with the cooling of the outer surface of the outer tube, unless, of course, the cooling medium can be circulated between the potential sheath and the resistor tube.

A very close approximation to the ideal condition can be obtained, however, by using only three or four pairs of potential leads placed symmetrically around the axis of the shunt, and such an arrangement does not interfere appreciably with the cooling of the resistor. This principle of using a number of symmetrically located potential leads in parallel gives the inductance an independence not only from external magnetic fields, but also from the internal fields caused by eccentricity of the current-carrying tubes. Thus, while a tubular shunt of radius $c \mathrm{~cm}$ with a single pair of potential leads may have its inductance changed by $2 q / c$ millimicrohenries per centimeter length by a displacement of $q \mathrm{~cm}$ between the axes, the use of four pairs of leads spaced $90^{\circ}$ apart reduces this change to only $3 q^{4} / c^{4}$ millimicrohenries per centimeter. Furthermore, measurements made on the apparent inductance of the shunt, when the different pairs of leads are used separately, give a basis for estimating the amount and direction of the effective eccentricity in the current distribution. If $L_{1}, L_{2}, L_{3}$, and $L_{4}$ are the observed inductances, then

$$
q=\frac{\sqrt{\left(L_{1}-L_{3}\right)^{2}+\left(L_{2}-L_{4}\right)^{2}}}{4 c}
$$

and

$$
\tan \alpha_{1}=\left(L_{2}-L_{4}\right) /\left(L_{1}-L_{3}\right)
$$

${ }_{16}$ Paterson and Rayner, Inst. Elec. Eng. J., 42, p. 455; 1909. 
where $\alpha_{1}$ is the angle between the line joining the axes of the two tubes and the line joining the axis of the outer tube to potential lead 1 , both lines being taken as lying in a plane normal to the axes.

It is, of course, not possible to locate the points of attachment of all the potential leads, which are grouped near one end of the shunt, on exactly the same equipotential surface. For this reason it is well to insert a resistance of a few tenths of an ohm in each potential lead, so that the currents circulating in the leads will be small. If the impedances in the various pairs of potential leads are equal, each pair will have equal weight in determining the potentials of the binding posts and the effective impedance of the shunt will be equal to the mean of the values observed by using the pairs separately. Any variation in the distribution of current over the current terminals of the shunt usually will affect differently the voltage drop between different potential leads, so that the mean value obtained by using a number of pairs of leads in multiple will be less affected by such sources of error. The resistance of the potential circuit is, of course, somewhat increased if these equalizing resistances are inserted, but for most purposes this resistance is not objectionable.

\section{SKIN EFFECT}

In normal laboratory practice, standards of resistance are intercompared and maintained by bridge measurements using direct current, and such methods are used to determine the resistance even of the standards which are to be used with alternating currents. It is therefore of importance in the design or use of alternating-current resistance standards to make sure that the effective resistance of the standard to alternating current of the frequency used does not differ from its direct-current resistance by a significant amount.

It can be shown by general dimensional reasoning that if the alternating-current resistance per unit length of a conductor depends only on the size and shape of the conductor, its resistivity $\rho$, its permeability $\mu$ and the frequency $f$ of the current, then the ratio of the alternating-current to the direct-current resistance must be a function of the dimensionless parameter $\frac{\mu f D^{2}}{\rho}$, where $D$ is some linear dimension of the cross section of the conductor. This principle has been stated more definitely by Dwight ${ }^{17}$ for the case of nonmagnetic material, as follows: "In any conductor or combination of conductors or in any return circuit, of a certain proportionate shape, a given value of $\frac{f}{R}$ corresponds to a certain value of $\frac{R^{\prime}}{R}$." Here $R^{\prime}$ and $R$ are the alternating-current and direct-current resistances per unit length, respectively. A more mathematical proof of this principle has been given by Slepian ${ }^{18}$ in his discussion of Dwight's paper.

Since manganin has a resistivity about twenty-five times that of copper, the skin effect in a structure of this material is the same as that in a similar structure of copper either having its transverse dimensions one-fifth as great or at one twenty-fifth the frequency and with the same dimensions. It is evident from this that the skin 
effect in a resistance standard will usually be relatively small, but, on the other hand, the accuracy needed in resistance standards is much greater than that considered in engineering work with copper conductors.

If the shunt has the form of a return circuit formed of two thin, wide conducting strips in parallel planes placed close together, the lines of magnetic force over the central portion of the structure are parallel to the width of the strip. The intensity of the field at the center is $4 \pi$ times the current per unit width of strip, and the intensity of the field at the outer surface of either strip and at points outside is zero. The emf induced by the alternation of a field having this distribution tends to make the current crowd toward the inner surfaces of each strip. The magnitude of this effect has been calculated by various writers for this case and for the analogous case of flux distribution in an iron lamination.

For low frequencies the ratio of the alternating-current resistance $R^{\prime}$ to the direct-current resistance $R$ can be expresssed as a power series in terms of the dimensionless parameter $m d$; where $d$ is the thickness of the strip in $\mathrm{cm}$ and $m$ is defined by

$$
m^{2}=\frac{8 \pi^{2} f}{\rho}=\frac{4 \pi \omega}{\rho}
$$

where $\rho$ is the resistivity in cgs units $\left(10^{-9} \mathrm{ohm}-\mathrm{cm}\right)$ and $f$ is the frequency in cycles per second. For copper at 60 cycles $m=1.65$ while for manganin at this frequency $m=0.33$.

For the case of two strips close together and so wide that the effect of their edges is negligible the calculations ${ }^{19}$ give for the skin-effect ratio

$$
\frac{R^{\prime}}{R}=1+\frac{m^{4} d^{4}}{45}-\frac{m^{8} d^{8}}{4,725}
$$

If, however, the strips are separated so far that they exert no appreciable electromagnetic effect on each other, there is a tendency for the current to crowd toward the edges of the strips as well as to the flat surfaces. This additional effect is much larger than that given by equation (20). This case has also been analyzed mathematically by Dwight, ${ }^{20}$ who obtains for low frequencies the equation

$$
\frac{R^{\prime}}{R}=1+0.0087432 p^{4}-0.000384 p^{8}+0.0000189 p^{12}
$$

where $p^{2}=\frac{m^{2} d w}{\pi}, w$ being the width of the strip.

When the strips are separated by a moderate distance, the mathematical calculations, while still, perhaps, possible by Dwight's method, would certainly be very laborious. This problem has been attacked experimentally by Kennelly, Laws, and Pierce, ${ }^{21}$ who have made an extensive series of measurements on the skin effect in conductors of a variety of shapes. Their data on copper strips $1 / 16$ inch thick and $1 / 2$,

${ }^{19}$ H. B. Dwight, Elec. Jn., 13, p. 157; 1916.

20 H. B. Dwight, Trans. A. I. E. E., 37, pt. 2, p. 1397; 1918.

21 Experimental researches on skin effect in conductors, Kennelly, A. E., Laws, F. A., and Pierce, P. H., Trans. A. I. E. E., 34, pt. 2, p. 1953; 1916. 
1 , and $1 \frac{1}{2}$ inches wide at frequencies up to 5,000 cycles indicate that equation (21) correctly represents the result of the tendency of the current to concentrate in the edges of the strip, but that even with conductors twenty-four times as wide as they are thick and at a spacing of only one-third of their thickness this edge effect is still so great at 4,000 cycles as to give a skin effect ratio of 1.4 instead of the value 1.04 computed by equation (20).

It is difficult to extrapolate the results of Kennelly, Laws, and Pierce to smaller values of the parameter $m$. Consequently, a number of measurements were made at the Bureau of Standards on the resistance of an experimental manganin ${ }^{22}$ shunt at frequencies up to 1,400 cycles per second, in order to obtain an indication of the magnitude of the resistance changes to be expected in this range.

The experimental shunt consisted of two strips $10 \mathrm{~cm}$ wide by $0.2 \mathrm{~cm}$ thick. These were joined to each other at one end and were

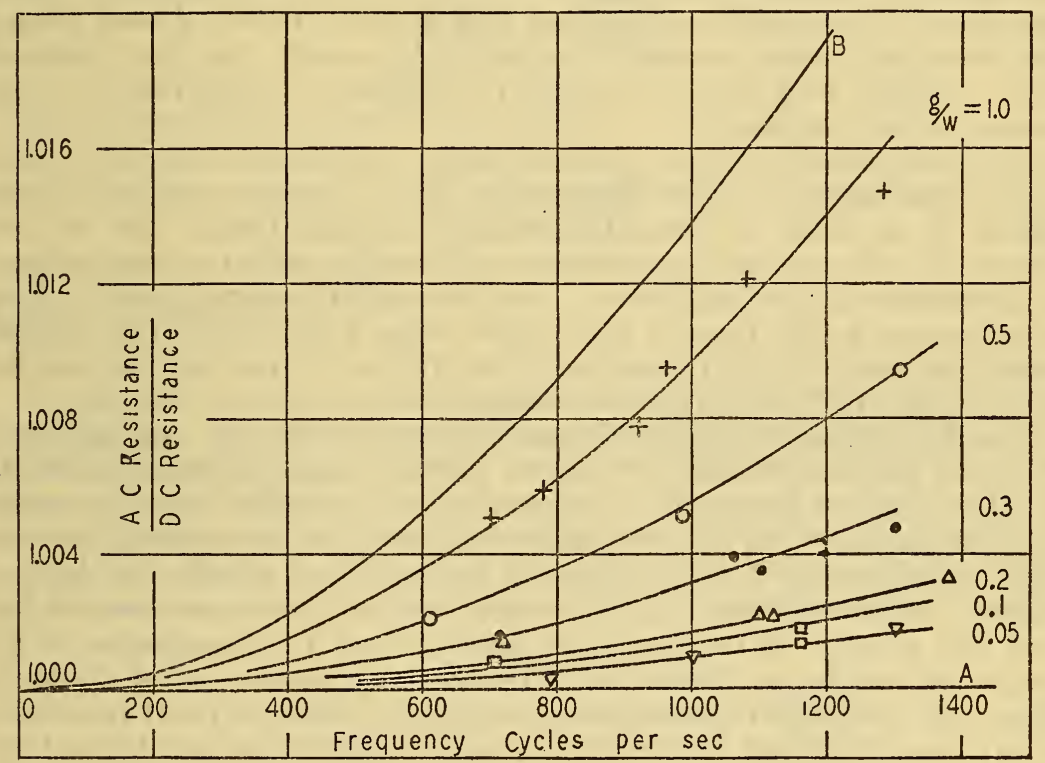

FIGURE 5.-Ratio of a. c. resistance to d. c. resistance as a function of frequency, as observed on manganin strips $2 \mathrm{~mm}$ thick and $10 \mathrm{~cm}$ wide at various spacings

Curve $O B$ shows the theoretical value for infinite spacing and curve $O A$ for zero spacing. Intermediate curves correspond to the values of the ratio of spacing $g$ to width $w$ marked opposite each curve.

provided with current terminals at the other. The total length from the current binding posts to the closed end was about $100 \mathrm{~cm}$, and the potential taps were located one on each strip $70 \mathrm{~cm}$ from the closed end. The spacing could be adjusted from a maximum value of $10 \mathrm{~cm}$ to a minimum of $0.5 \mathrm{~cm}$.

22 Unfortunately, this shunt (which had been designed for another purpose) included at its closed end a section of sheet copper $15 \mathrm{~cm}$ long, $10 \mathrm{~cm}$ wide, and $0.022 \mathrm{~cm}$ thick. This piece of copper was sufficiently flexible to permit of ready adjustment of the spacing between the manganin strips, but it contributed about $0.00012 \mathrm{ohm}$ to the total resistance of the shunt, which was about $0.00247 \mathrm{ohm}$ for direct currents. The measuring current was maintained quite definitely at 80 amperes in all the measurements, but variations in the temperature of the copper considerably affected the precision of the results, and may have caused the skin effect to be slightly greater than that which would occur in a similar structure made entirely of manganin. 
The resistance of this shunt was compared with that of a tubular shunt also of manganin. This latter shunt had a resistance of $0.0009285 \mathrm{ohm}$, the diameter of the tube being $5.1 \mathrm{~cm}$ and the thickness $0.15 \mathrm{~cm}$. It had, of course, no edge effect, and its change in resistance with frequency as computed from the formulas given below was entirely negligible. The results are plotted in Figure 5, the ordinates being the ratio of alternating current to direct-current resistance while the abscissas are frequency. The curves $O A$ and $O B$, of which $O A$ practically coincides with the axis of frequency, are computed from equations (20) and (21), respectively. The observed points are indicated by crosses, and it will be seen that the increase in resistance with frequency at each spacing follows the same general trend as the curve $O B$ corresponding to an infinite spacing, though to a less extent. Thus, the change in resistance at $10 \mathrm{~cm}$ spacing is about 60 per cent, while that for the $1 / 2 \mathrm{~cm}$ spacing is 6 per cent of the change computed for infinite spacing. For the frequencies used equation (21) is rapidly convergent and the solid curves drawn among the observed points actually correspond to only the first term of such a series and show a change of resistance proportional to the square of the frequency.

The inaccuracies in the measurements somewhat mask the trend of the resistance at lower frequencies, but it appears probable from Figure 5, as well as from theoretical considerations, that at low frequencies the increase in resistance still may be taken as proportional to the square of the frequency. On this basis the shunt used in these experiments would have a skin effect ratio at 60 cycles per second, exceeding unity by 350 parts per 1,000,000 at $10 \mathrm{~cm}$ spacing and by 50 parts per $1,000,000$ at $1 \mathrm{~cm}$ spacing.

The data shown in Figure 5 were obtained with only one thickness $(0.2 \mathrm{~cm})$ and one width $(10 \mathrm{~cm})$ of strip. Since the ratio of width to thickness is so great $(50: 1)$, however, it is probable that the results are representative of the skin effect in any thin strip which has the same resistance per unit length as the strip on which the data of Figure 5 were obtained. This assumption and the experimental fact that the change in resistance is proportional to the square of the frequency can be combined with Dwight's principle stated above to show that the ratio of alternating current to direct-current resistance of any circuit formed of two thin strips at a spacing of $g / w$ times their width is given for any frequency $f$, thickness $d$, and resistivity $\rho$ by the equation

$$
\frac{R^{\prime}}{R}=1+\frac{f^{2} d^{2} w^{2}}{\rho^{2}} \cdot \frac{\rho_{0}^{2}}{f_{0}^{2} d^{2}{ }_{0} w_{0}^{2}}\left(F_{0}(g / w)-1\right)
$$

where $F_{0}(g / w)$ is the ratio of alternating-current to direct-current resistance given in Figure 5 for the same ratio giw of spacing to width and for $d_{0}=0.2 \mathrm{~cm}, w_{0}=10 \mathrm{~cm}, \rho_{0}=38,600 \mathrm{c} . \mathrm{g}$. s. units, and for the value of $f_{0}$ indicated by the abscissa.

Equation (22) is, of course, applicable only at low frequencies where the parameter $p$ (equation (21)) is less than 0.5 and for strips so thin that the concentration of current at the edges is the predominating effect (that is, perhaps $d / w \equiv 0.2$ ).

As an example of the use of equation (22) consider the skin effect at 60 cycles for a circuit consisting of two copper strips $25 \mathrm{~cm}$ wide 
and $0.5 \mathrm{~cm}$ thick, spaced $10 \mathrm{~cm}$ center to center. Taking $\rho=1,750$ c. g. s. units, the factor $\frac{f^{2} d^{2} w^{2}}{\rho^{2}}$ is 0.184 . The ratio of spacing to width is $g / w=0.4$ and from Figure 5 for $f_{0}=1,200$ cycles we get by interpolating between the curves, $F_{0}(g / w)=1.0063$, while the factor $\frac{\rho_{0}{ }^{2}}{f_{0}{ }^{2} d_{0}{ }^{2} w_{0}{ }^{2}}$ is 258 . Hence, the skin-effect ratio for the copper strap is $1+0.184 \times 258 \times 0.0063=1.030$.

The skin effect in shunts of the tubular type can be computed mathematically. The case of a circular wire or tube with the return circuit at a great distance has been treated by a number of writers. ${ }^{23}$ For low frequencies the ratio of alternating-current to direct-current resistance can be expressed by the first two terms of a power series, as follows:

For a solid wire of radius $a$

$$
R^{\prime} / R=1+\frac{m^{4} a^{4}}{192}
$$

For a thin-walled tube of thickness $d$

$$
R^{\prime} / R=1+\frac{m^{4} d^{4}}{45}
$$

where as before $m^{2}=\frac{8 \pi^{2} f}{\rho}$

When the current-carrying circuit consists of two concentric tubes, the situation is more complicated. Russell ${ }^{24}$ has published a very thorough study of the effective resistance and inductance of a concentric main, developing formulas both for low and for high frequencies. His equations can be still further simplified if we limit ourselves to low frequencies and to cases where the walls of the tubes are thin in comparison with their diameter. For this case Russell's equation (101) reduces to

$$
R^{\prime}=\frac{\rho}{\pi a^{2}}\left(1+\frac{m^{4} a^{4}}{192}\right)+\frac{\rho}{\pi\left(c^{2}-b^{2}\right)}\left(1+\frac{m^{4} d^{4}}{45}\right)
$$

where $R^{\prime}$ is the effective alternating-current resistance per unit length of the concentric main which is formed of a tube having outer and inner radii $c$ and $b$, respectively, and a coaxial return conductor in the form of a rod of radius $a$. As before $d=c-b$. When the inner conductor is also a thin-walled tube of thickness $d_{1}$, Russell's equations (113) and (114) give

$$
R^{\prime}=\frac{\rho}{\pi\left(c^{2}-b^{2}\right)}\left(1+\frac{m^{4} d^{4}}{45}\right)+\frac{\rho}{2 \pi\left(a d_{1}-\frac{d_{1}^{2}}{2}\right)}\left(1+\frac{m^{4} d_{1}^{4}}{45}\right)
$$

${ }^{23}$ See bibliography in paper by Kennelly, Laws, and Pierce, referred to in footnote 21.

24 A. Russell, The Effective Resistance and Inductance of a Concentric Main and Methods of Computing the Ber and Bei and Allied Functions, Phil. Mag., 6th series, 17, p. 524; 1909. 
Considerable caution must be exercised in passing from these cases to those in which the potential leads span a part of only the outer or of the inner conductor and are located entirely outside the outer tube or inside the inner tube.

The difficulty in separating the potential drops in the two conductors arises from the fact that the outer tube, if it has a finite thickness, receives power by transformer action from the inner conductor. Hence, its mere presence in the magnetic field of the inner conductor would increase the power drawn from the source even if the outer tube were not in metallic contact with the alternating-current circuit. The total resistance given by equation (25) or equation (26) must be considered as that quantity which, when multiplied by the square of the total current, gives the total power consumption. An integration of the quantity $i^{2} \rho d v$ over the cross section of the inner conductor, using for the current density $i$ the value given in Russell's equation (65), shows that the power dissipated as heat in the inner conductor per unit length is given by

$$
H_{1}=I^{2} \frac{\rho}{\pi a^{2}}\left(1+\frac{m^{4} a^{4}}{192}\right)
$$

Hence, the heat developed in the outer conductor must be

$$
H_{2}=\frac{I^{2} \rho}{\pi\left(c^{2}-b^{2}\right)}\left(1+\frac{m^{4} d^{4}}{45}\right)
$$

The potential drop in unit length of the outer conductor can be evaluated either from Russell's equation (78) by taking $i^{\prime} \rho$ for $r=c$ and substituting the proper values for the constants $A, B, C, D$, and $i_{0}$, or by a direct solution of the differential equations in terms of Bessel functions to which Taylor's theorem can then be applied. The result by either method gives for the component of the voltage drop in phase with the current

$$
E=\frac{\rho I}{\pi\left(c^{2}-b^{2}\right)}\left(1-\frac{7}{360} m^{4} d^{4}\right)
$$

This indicates that such a shunt will actually have less effective resistance to alternating than to direct currents, but this is quite consistent with the fact that the current tends to crowd toward the inner surface of the outer tube.

Multiplying equation (29) by the current and subtracting the result from equation (28) gives as the power transferred from the inner to the outer conductor by transformer action

$$
P=I^{2} \frac{\rho}{\pi\left(c^{2}-b^{2}\right)} \frac{m^{4} d^{4}}{24}
$$

Adding this to equation (27) and dividing by the square of the current gives for the resistance of the inner conductor as measured with potential leads brought out radially through holes in the outer tube

$$
R_{i}=\frac{\rho}{\pi a^{2}}\left(1+\frac{m^{4} a^{4}}{192}\right)+\frac{\rho}{\pi\left(c^{2}-b^{2}\right)} \frac{m^{4} d^{4}}{24}
$$


B. S. Journal of Research. RP133

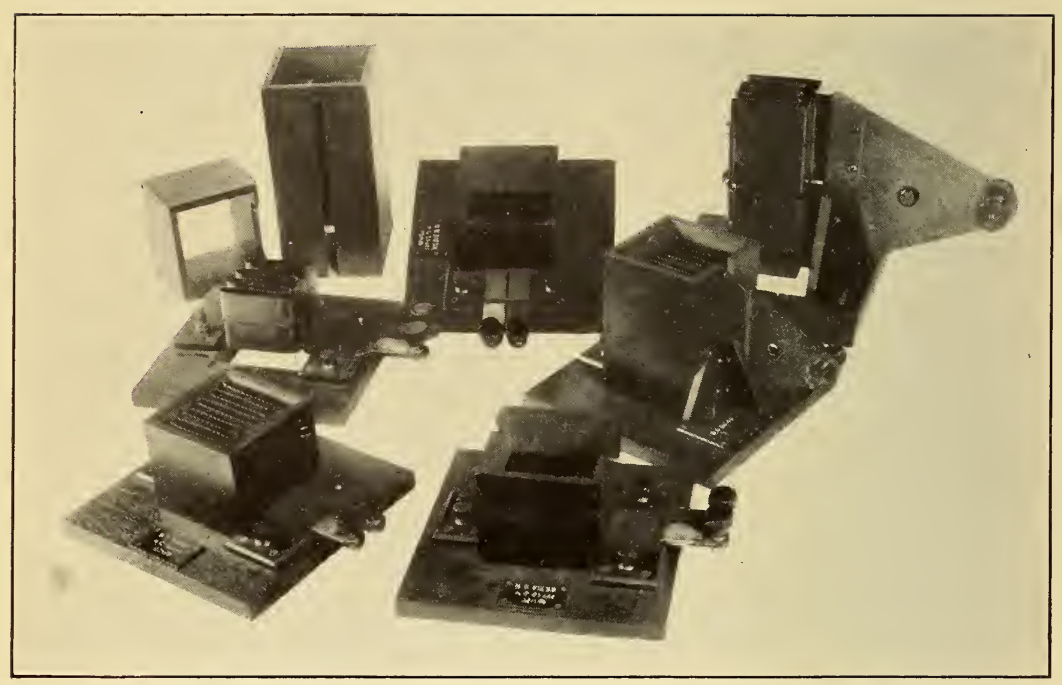

FIGURE 6.-Group of air-cooled shunts ranging in resistance from 0.05 to 0.001 ohm and in current capacity from 10 to 500 amperes 
If, however, the potential leads are brought out through the hollow center of a tubular inner conductor we have

$$
R_{i}=\frac{\rho}{2 \pi\left(a d_{1}-\frac{d_{1}^{2}}{2}\right)}\left(1-\frac{7 m^{4} d_{1}^{4}}{360}\right)
$$

where $d_{1}$ is the thickness of the inner conductor.

It is interesting to note that equation (28) indicates that for the case of two tubes of equal thickness and both so large as to have nearly the same radius the ratio of alternating-current to direct-current resistance approaches the same value as that given in equation (20) for infinitely wide strips.

\section{AIR-COOLED RESISTANCE STANDARDS}

As examples of the application of the principles of design which have been discussed in the preceding sections we may consider the two groups of alternating-current resistance standards which were constructed at the Bureau of Standards for use in the current transformer testing. The first group of shunts, built in 1923 by F. M. Defandorf and R. D. Wyckoff, range in resistance from 0.05 to $0.001 \mathrm{ohm}$ and are designed for a voltage drop of 0.5 volt. Figure 6 shows the general appearance of these shunts, and Figure 7 shows the arrangement of potential leads, etc., in a typical shunt of this group. Table 1 gives the essential dimensions and other data.

TABLE 1.-Data on air-cooled shunts

\begin{tabular}{|c|c|c|c|c|c|c|}
\hline Resistance & 0.05 & 0.02 & 0.01 & 0.005 & 0.0025 & 0.001 \\
\hline Current rating & 10 & 25 & 50 & 100 & 200 & 500 \\
\hline Length & 75 & 75 & 75 & & 76 & 72 \\
\hline Width & 1.6 & 4. 0 & 8.0 & 7.0 & 13.3 & 20.0 \\
\hline Thickness & .056 & .056 & .056 & .081 & .10 & .14 \\
\hline Watts per square centimeter at rated cur- & & & & & & \\
\hline $\begin{array}{l}\text { rent } \\
\text { Phase angle at } 60 \text { cycles, observed }\end{array}$ & .021 & .021 & .021 & .055 & .050 & .083 \\
\hline _. minutes.. & +.4 & +.3 & +.1 & -.3 & -.4 & -2.0 \\
\hline
\end{tabular}

The cooling of these shunts is effected by placing them in an air stream which is supplied by a motor-driven blower. The air is led from the blower through a hole in the table top and passes up through a wooden channel 11 by $11 \mathrm{~cm}$, inside of which the shunt proper is located. The air velocity at full speed of the blower is about 1,600 $\mathrm{cm}$ per second ( 36 miles per hour), and the heat dissipated is 0.017 watt per square centimeter per degree centigrade temperature rise. Consequently, the temperature rise of the 500 ampere shunt at fullrated current is only $5^{\circ} \mathrm{C}$., and that of the other shunts is considerably less. The cooling was found to be roughly proportional to the square root of the air velocity. This method of cooling avoids the risk of corrosion of the shunt metal by contact with oil which has become acid and eliminates the spilling and leakage usually associated with oil-cooled apparatus. The noise made by the blower is slightly objectionable at the highest speeds required to cool the largest shunts. The cooling surface required to transfer larger amounts of power than the greatest here used ( 250 watts) makes the design of air-cooled alternating-current shunts for higher ratings rather difficult, and the oil- 
cooled shunts described below have been developed for higher current ranges.

While the general principle of compensating the inductance of shunts by suitably locating the potential leads has long been recognized, ${ }^{25}$ most of the designs embodying this principle have involved

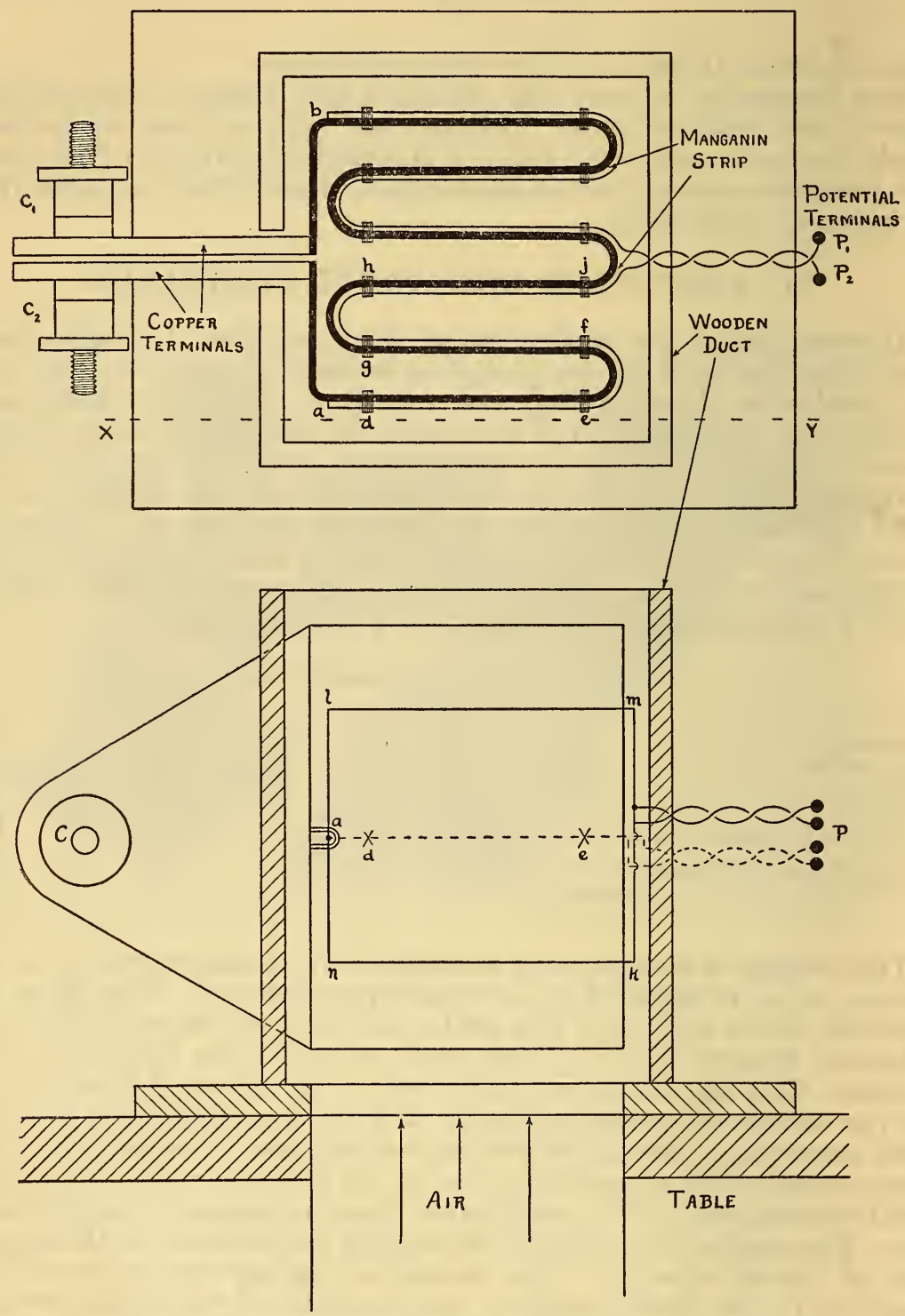

Figure 7.-Plan and cross section of air-cooled shunt

the use of a potential sheath which has covered one side of the working resistor, and thus has materially obstructed the available cooling surface. In this group of air-cooled shunts all possible cooling surface 
is needed, and the current-carrying strips are therefore placed about $9 \mathrm{~mm}$ apart, so that both sides are exposed to the cooling air stream. The current circuit, therefore, produces a considerable magnetic field. The effect of this on the self-inductance of the shunt as a whole has been compensated by locating the potential leads as shown in Figure 7 . The ends of the leads are soldered to $U$-shaped lugs at $a$ and $b$, and each lead (of flattened No. 30 wire) is tied tightly against the manganin strip as at $d, e, f, g, h$, or $l, m \ldots \ldots$..... throughout its foldings to the center $(j)$ of its length, where the two leads meet and run as a twisted pair to the potential binding posts $p_{1} p_{2}$.

In the shunts of the lower four current ranges the potential leads are placed in the center of the width of the manganin strip, as indicated at de, Figure 7 . This gives a sufficiently close compensation for inductance and skin effect, and renders the shunt practically immune to stray magnetic fields. In the case of the 200-ampere shunt the lead is placed as indicated at $l m$, Figure 7, and in the 500-
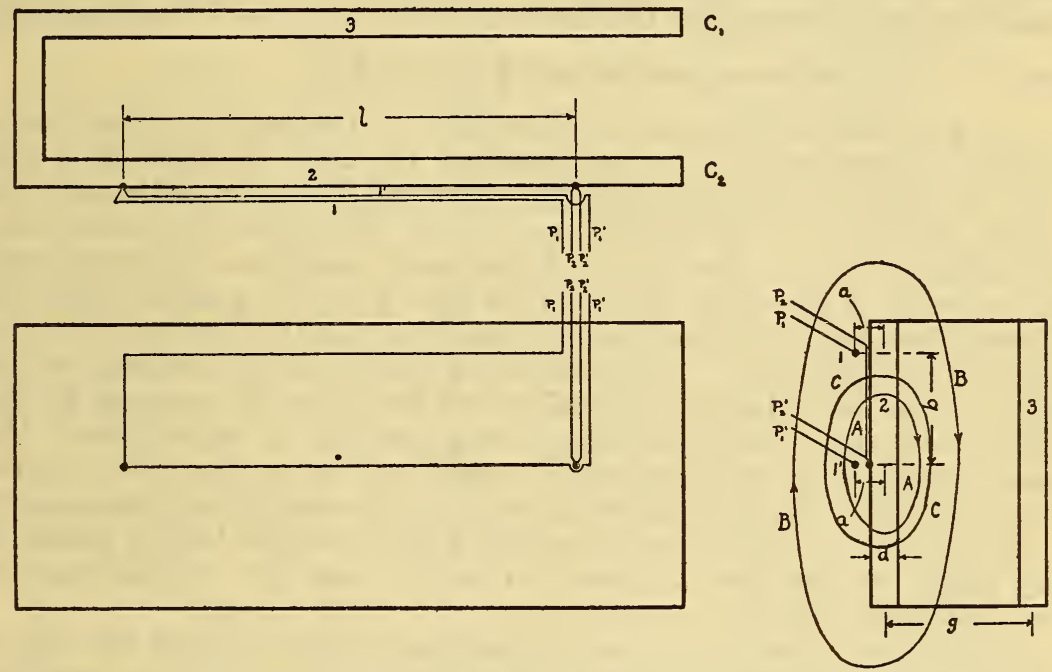

Figure 8.-Schematic diagram of simple shunt with alternative potential leads

ampere shunt two sets of potential leads connected in parallel are used as indicated at $l m$ and $n k$, Figure 7 . The placing of the leads down from the edge of the strip by a distance equal to two-tenths of the width of the strip has the effect as shown below of giving a more accurate compensation of inductance and skin effect, while the use of two leads in parallel restores the immunity to stray fields which is sacrificed by the unsymmetrical location of the single lead in the 200-ampere shunt.

The effect of the location of the potential leads in shunts of this type can be seen if we first consider the set of conductors shown in Figure 8. Assuming that the formula for long straight conductors is applicable, the 4-terminal inductance is given by

$$
\begin{aligned}
L & =2 l\left[\log _{e} \frac{D_{12} D_{23}}{D_{13} D_{22}}\right] \\
& =2 l\left[\log _{e} D_{12}-\log _{e} D_{22}+\log _{e} D_{23}-\log _{e} D_{13}\right]
\end{aligned}
$$


It will be seen that the first two terms are independent of the distance $g$ to the return lead, and that if the return were at a great distance; so that $\log _{e} D_{13}=\log _{e} D_{23}$, the inductance would be given by

$$
L=2 l\left[\log _{e} D_{12}-\log _{e} D_{22}\right]=2 l F_{1}
$$

If we limit ourselves for the present to the case of thin strips when $a$ and $d$ are zero, the equations $I(c)$ and $I I(d)$ given in the appendix show that the factor in the brackets is given by

$$
F_{1}=\frac{1}{2}+n \log _{e} n+(1-n) \log _{e}(1-n)
$$

where $n=\frac{1}{2}-\frac{b}{w}$. The quantity $F_{1}$ has the value +0.5 for $n=0$ or $n=1$ (that is, if the potential lead is run along either edge of the strip); it has a minimum value of -0.193 for $n=0.5$ (that is, if the potential lead is run along the center of the strip); and it has the value zero if $n=0.2$ or 0.8 approximately $\left(\frac{b}{w}= \pm 0.3\right)$.

The physical significance of this result can be seen by noting that a certain amount of flux as indicated by the lines of force $A-A$ and $C-C$ in Figure 8 cuts through the strip and is linked with only the center portion of it, although most of the flux links the entire strip as indicated by the line $B-B$. A potential lead placed near the axis of the strip is therefore linked by a flux which is greater than the average flux linking the entire strip, and the shunt as a whole shows a negative inductance. A potential lead placed along the edge of the strip, on the other hand, is linked by less flux than the average for the entire strip and the inductance compensation is incomplete. For some intermediate position, which works out to be about two-tenths of the width of the strip in from the edge, the compensation is exact.

In fact, the compensation is correct if the potential lead is placed at any point on that line of force $C-C$ which cuts the central plane of the strip at $b=0.3 w$.

If the return lead 3 is at only a moderate distance from the rest of the shunt, the distances $D_{13}$ and $D_{23}$ will not be exactly equal, and the 4-terminal inductance will contain a contribution

$$
2 l F_{2}=2 l\left[\log _{e} D_{23}-\log _{e} D_{13}\right]
$$

which will depend on the spacing $g$ between the strips. From the equations II $(a)$ and I $(a)$ given in the appendix it follows that for the case of thin strips

$$
\begin{aligned}
& F_{2}=\frac{1}{2} x^{2} \log _{e} x^{2}+\frac{\left(1-x^{2}\right)}{2} \log _{e}\left(1+x^{2}\right) \\
& -\frac{n}{2} \log _{e}\left(n^{2}+x^{2}\right)-\frac{(1-n)}{2} \log _{e}\left\{(1-n)^{2}+x^{2}\right\} \\
& +x\left\{2 \cot ^{-1} x-\cot ^{-1} \frac{x}{1-n}-\cot ^{-1} \frac{x}{n}\right\}-\frac{1}{2}
\end{aligned}
$$

where $x=\frac{g}{w}$. The function of $x$ and $n$ given by equation (37) is plotted in Figure 9 against $x$ for several values of $n$, and in Figure 10 against 
$n$ for several values of $x$. These curves show that as the return strip 3 starting at a great distance is brought closer and closer to the outgoing strip 2 the term $2 l F_{2}$ changes from zero in such a way as to tend to offset the inductance $2 l F_{1}$ computed from the $D_{12}$ and $D_{22}$ terms. This tendency to compensation exists whether the original inductance is positive or negative, and in the limit when the strips are in coincidence so that $g$ and $x$ are zero, the compensation becomes perfect (that is, $F_{2}(0, n)=-F_{1}(n)$ ). This, of course, is to be ex-

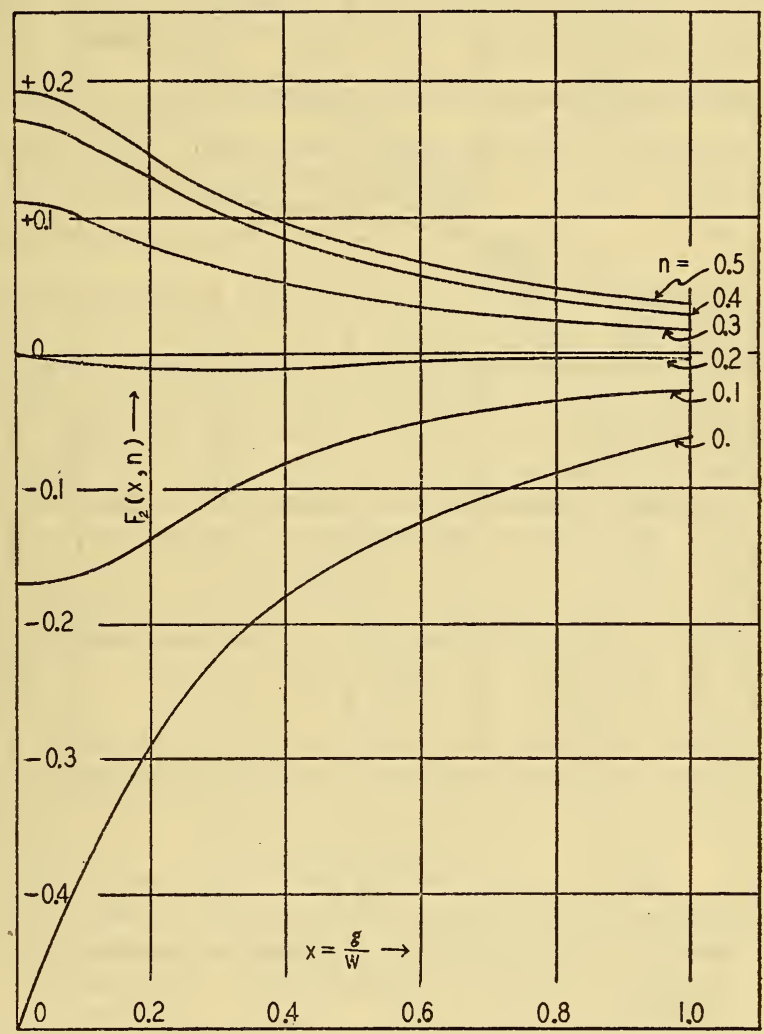

FigURE $9 .-F_{2}(x, n)=\frac{1}{2} x^{2} \log _{e} x^{2}+\frac{\left(1-x^{2}\right)}{2} \log _{e}\left(1+x^{2}\right)$ $\left.-\frac{n}{2} \log _{\mathrm{e}}\left(n^{2}+x^{2}\right)-\frac{(1-n)}{2} \log _{\Theta}\left\{(1-n)^{2}+x^{2}\right)\right\}+x\left\{2 \cot ^{-1} x\right.$ $\left.\left.-\cot ^{-1}\right) \frac{x}{1-n}-\cot ^{-1} \frac{x}{n}\right\}-\frac{1}{2}$ plotted as a function of $x$ for the values of $n$ marked on the curves

pected, since when the strips coincide the outgoing and return currents completely neutralize each other, element by element.

Figure 9 also shows, however, that in case $n=0.2$ or 0.8 the net effect of the return lead 3 is practically zero for all spacings. It is therefore evident that the location of the potential lead at either of these positions will cause complete compensation for the self-inductance of the current circuit and is especially desirable in designs, such as those described below, where the current-carrying parts form a zigzag path, 
so that each potential lead is subject to the magnetic effect from a number of current strips.

In the actual construction of a shunt the current-carrying strips must, of course, have a finite thickness. If the thickness of each is $d$, the inductance will be less than that given by equations (35) and

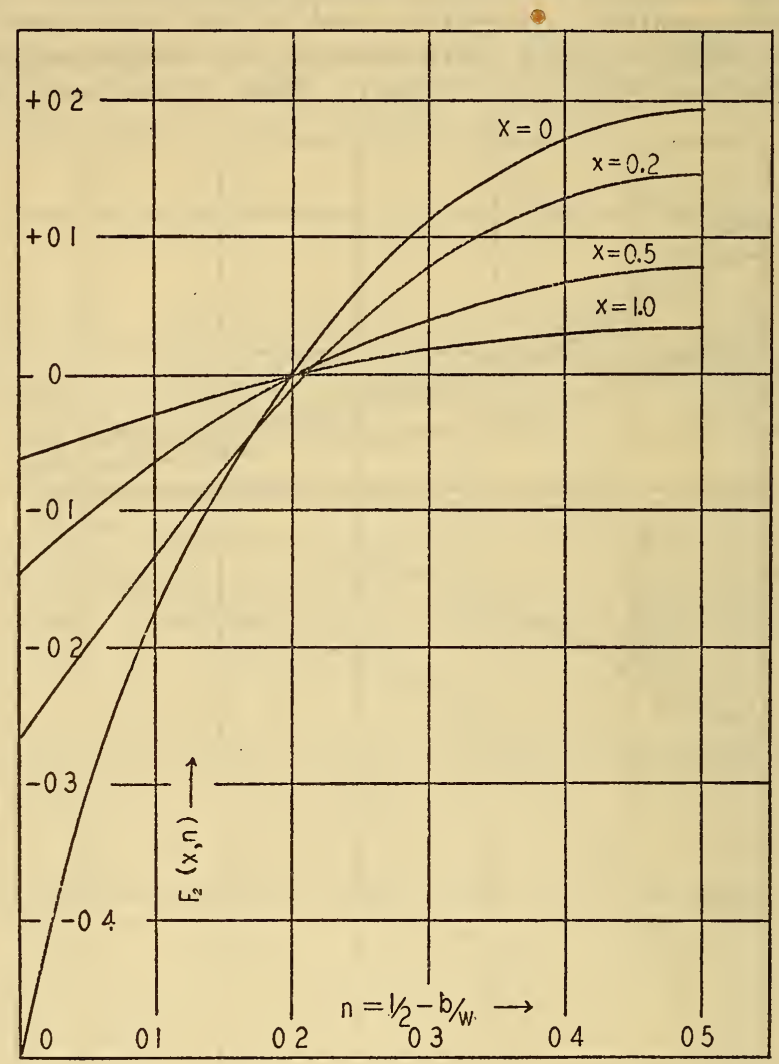

FIgURe 10.-Graph of $F_{2}(x, n)$ given in equation (37) plotted as a function of $n$ for the values of $x$ marked on the curves

(37). The correction resulting from the finite thickness is found from equations $I(c) ; I I(d), 2 ; I I(a)$ and $I(a)$ of the appendix to be

$$
\begin{aligned}
& 2 l F_{3}=22\left[-\frac{\pi}{3} \frac{d}{w}+\frac{d^{2}}{24 w^{2}}\left\{\frac{25}{3}-\frac{1}{n(1-n)}-4 \log _{e} \frac{d}{w}\right.\right. \\
& \left.\left.-2 \log _{e}\left(1+\frac{1}{x^{2}}\right)+\frac{n}{x^{2}+n^{2}}+\frac{(1-n)}{(1-n)^{2}+x^{2}}\right\}-\cdots\right]
\end{aligned}
$$

Inspection shows that for most values of $\frac{d}{w}$ and $\frac{g}{w}$ likely to occur the terms involving $\frac{d^{2}}{w^{2}}$ can be neglected unless $n$ is nearly zero or unity.

Similarly the potential lead can not well be made to lie exactly in the medial plane of the strip, but is at a small distance $a$ from this plane. In practice $a$ will be slightly greater than $\frac{d}{2}$ because of the 
insulation between lead and shunt. The effect of this spacing $a$ is to add to the inductance a contribution

$$
\begin{aligned}
& 2 l F_{4}=2 l\left[\frac{a}{w}\left\{\pi \mp \tan ^{-1} \frac{n}{x} \mp \tan ^{-1} \frac{(1-n)}{x}\right\}\right. \\
& \left.+\frac{a^{2}}{2 w^{2}}\left\{\frac{\left(x^{2}+n-n^{2}\right)}{\left(x^{2}+n^{2}\right)\left(x^{2}+(1-n)^{2}\right)}-\frac{1}{n(1-n)}\right\}--\right]
\end{aligned}
$$

The upper signs apply when the potential lead and the return conductor lie on opposite sides of the strip, the lower signs when they are on the same side. Here, again, the terms in $\frac{a^{2}}{w^{2}}$ are negligible under all usual conditions. For close spacings (small values of $g$ or $x$ ) the first-order term also becomes small while for large values of $g$ or $x$ it approaches the value $2 \pi l \frac{a}{w}$.

In deducing equation (39) from the basic equation (33), the term $\log _{e} D_{12}$ has been taken from equation $I(c)$ in the appendix. The value of $\log _{e} D_{13}$ is obtained by putting $(g+a)$ in place of $a$ in the general equation $I(a)$ and then expanding for $a$ small.

In the arrangement actually used in the air-cooled shunts it will be seen from Figure 7 that each stretch of manganin strip and its part of the potential lead correspond to conductors 2 and 1 , respectively, in Figure 8. In place of a single-return conductor as 3 in Figure 8 there is a succession of conductors which are spaced at consecutively greater and greater distances from conductor 2 and which carry currents which flow alternately in opposite directions. The inductance can, therefore, be estimated by adding to the result of equation (34) a succession of contributions computed by the use of equation (36) for the various other conductors. This procedure, of course, does not make proper allowance for the connections across the ends, but phase angles thus computed have been found in most cases to agree with experimentally observed values to within one minute at 60 cycles, after the corrections $2 l F_{3}$ and $2 l F_{4}$ have been applied.

It is evident from the foregoing paragraphs that this design offers a convenient means of obtaining a shunt of small and definite inductance. In the case of manganim shunts having a resistance of $0.01 \mathrm{ohm}$ or more the correction terms $2 l F_{3}$ and $2 l F_{4}$ may be of the same order as the main terms $2 l F_{1}$ and $2 l F_{2}$, but the total phase angle is so small that a satisfactory design is obtained by running the potential lead at the center of the width of the shunt. With shunts of lower resistance made with wider and thicker strips the phase angle becomes of greater importance and it becomes more desirable to move the potential lead nearer to the edge of the strip to maintain sufficientiy close compensation. Because of uncertainty in the distance $a$ between potential lead and strip it is usually necessary to determine the exact value of inductance experimentally by comparison with a more readily computable standard.

Any theoretical estimate of the skin effect in shunts of this type is very difficult because of the fact that the potential lead is placed so close to the current-carrying parts that it is subject to a greater inductive action from some parts of the current than from others.

A general idea of the apparent resistance to be expected with such a construction can be seen from the following considerations. In 
the case of a thin strip conductor with a return conductor at a moderate spacing the current density existing near the edges of the strip tends to lead in phase as compared with the total current, and also to be somewhat greater in magnitude than the average current density over the entire cross section. This is because the edges are linked by less than the average amount of magnetic flux. Conversely, the current density in the central portion of the strip lags in time phase behind the total current. Now, the total electromotive force induced in a potential lead, which is located, say, along the edge of such a strip, may be considered as the sum of contributions from elements of current flowing in all parts of the strip. Each contribution of emf is strictly in time quadrature with the element of current which produces it and is proportional in magnitude to the mutual inductance between the current element in question and the potential lead. This mutual inductance will be greatest, of course, in the case of those elements located nearest to the potential lead and, therefore, these elements of current (in this case those near one edge of the strip) will have more weight than the rest in determining the time phase of the total emf induced. The result is that the induced emf leads the quadrature position and has a component in phase with the current so that the total effective resistance of the 4-terminal conductor is greater than it would be if there were no inductive action. Conversely, a shunt having its potential lead placed near the center of the strip has an apparent negative skin effect. Furthermore, it would be expected that if the potential lead were placed in an intermediate location, so that the current elements near it had substantially the same phase as the total current, then the 4-terminal conductor would show only the normal skin effect corresponding to the actual increased power dissipation in the metal.

As a check upon the foregoing analysis, a series of measurements were made on the effective resistance, at frequencies of about 60,600 , and 1,000 cycles per second, of an experimental shunt which consisted of two parallel straight strips which formed the outgoing and return conductors. A number of pairs of potential leads were clamped against the outside of one of the strips at various distances from its axis. For convenience the points of contact between the potential leads and the strip were common to all the pairs of leads and were at two points on the center of the strip. Since the equipotential surfaces are necessarily planes perpendicular to the axis of the shunt the same results would have been obtained if the points of contact had been distributed along two such planes. The strip was of manganin $10 \mathrm{~cm}$. wide and $0.2 \mathrm{~cm}$. thick. The return circuit was formed of a similar straight strip which could be placed at various distances from the working strip but which carried no potential leads. The results are shown in Figure 11. Here the abscissas are the distance of the potential lead from the edge of the strip, expressed in terms of the width of the strip. The ordinates are the increase of the alternating-current over the direct-current resistance, divided by the direct-current resistance and by the square of the frequency, for the 4-terminal conductor formed by the strip and the potential lead placed as indicated by the abscissa. The several curves shown correspond to different spacings of the return conductor. The number on each curve is the ratio of the spacing between the strips to the width of the strip. Figure 11 is based mainly on data obtained with a fre- 
quency of 1,000 cycles, but results at other frequencies showed the change in resistance to be roughly proportional to the square of the frequency.

It will be seen that the general trend of the curves is in accordance with what would be expected from the theoretical considerations previously discussed. The shunt when used with potential leads at or near the center of its width shows a very considerable decrease in resistance with increase of frequency, while if the potential lead lies

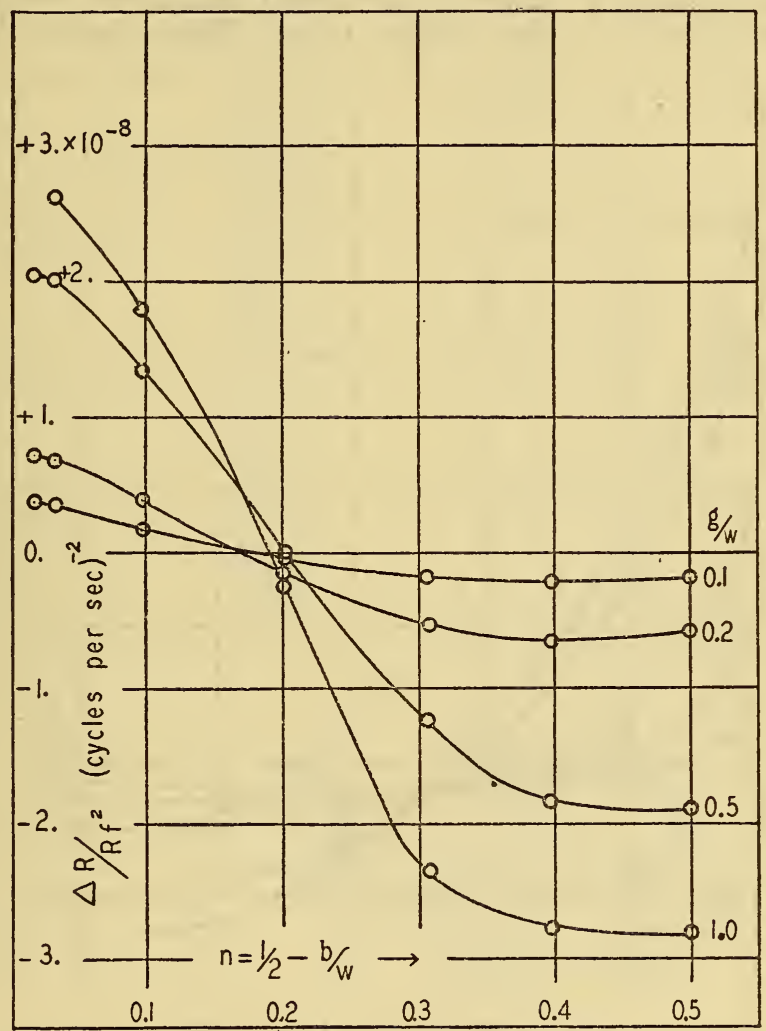

Figure 11.-Observed relation between the four-terminal a. c. resistance of a flat-strip shunt and the location of the potential leads fastened tightly against the strip

The abscissas are the distance measured parallel to the width of the strip from its edge to the potential lead, expressed in terms of the width of the strip. The ordinates are the increase in resistance from d. c. to a. c. divided by the d. c. resistance and by the square of the frequency. The number marked opposite each curve indicates the ratio of the spacing of the strips $g$ to their width $w$.

near the edge the skin effect is positive. The position which gives zero net skin effect for the shunt is substantially the same as that corresponding to zero effective inductance and varies only slightly with the spacing to the return conductor.

Inductance measurements were also made on the experimental strip shunt as a check upon the theoretical equations. Figure 12 shows by the curves the net effective inductance per unit length of the experimental shunt as computed from equations (35) and (37) after correct- 
ing for the thickness of the strip by equation (38) and for the location of the potential lead by (39). The plotted points show the values observed experimentally at 60 cycles. The agreement is quite satisfactory, and the small discrepancies can reasonably be attributed to uncertainty in the distance between the strip and the potential lead.

\section{OIL-COOLED RESISTANCE STANDARDS}

The second group of alternating-current resistance standards consists of two shunts of the tubular type. The detailed design and

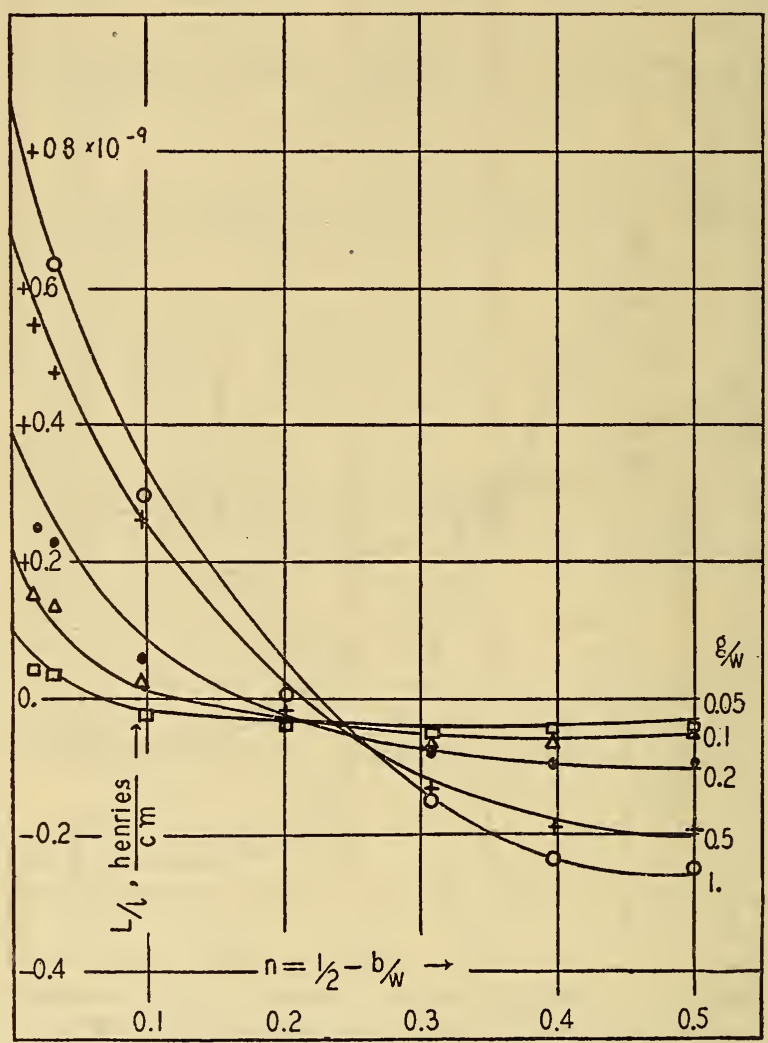

FIGURE 12.-Four-terminal inductance per centimeter length of a flat strip shunt as a function of the distance of the potential lead from the edge of the strip expressed in terms of the width of the strip

The number marked opposite each curve indicates the ratio of the spacing of the strips $g$ to their width $w$.

adjustment of these shunts were worked out by R. D. Wyckoff and R. L. Smith. The mechanical construction was done by J. M. S. Kaufman. The shunts were completed early in 1929. One is rated at 1,000 amperes, $0.0005 \mathrm{ohm}$, and the other has a current rating of 2,500 amperes and has two sets of potential terminals corresponding to resistances of 0.00025 and $0.0002 \mathrm{ohm}$. The considerable amount of power dissipated as heat in these shunts makes the use of oil cooling desirable. The tubular construction was chosen as offering at once 
convenient guiding surfaces for the cooling oil, freedom from any tendency to produce magnetic field at external points and the possibility of obtaining the inductance by computation without requiring comparison with any other shunt. Figures 13 and 14 show the general

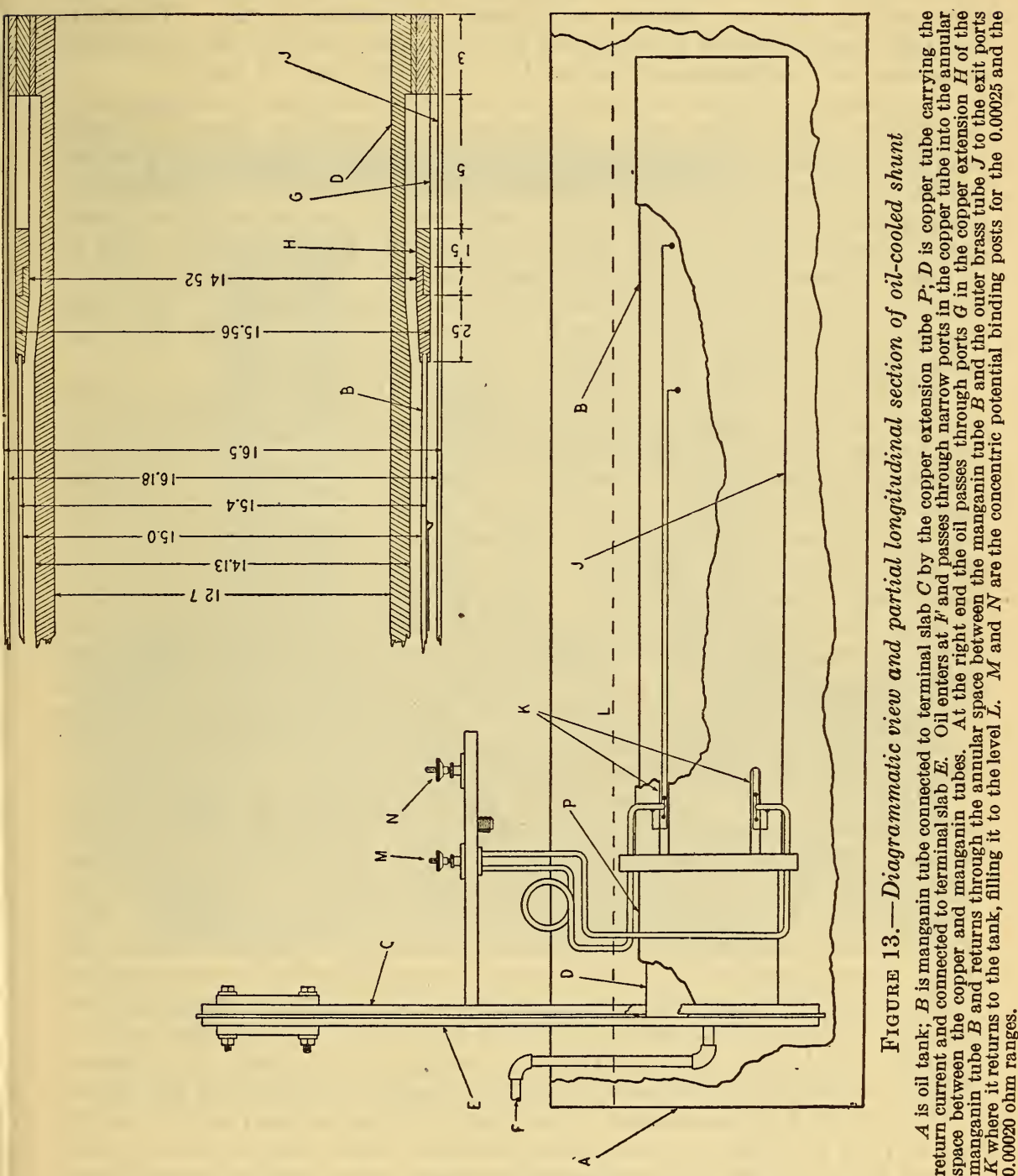

appearance of these shunts, and Table 2 gives the essential data concerning them.

TABLE 2.-Data on oil-cooled shunts

\begin{tabular}{|c|c|c|c|}
\hline $\begin{array}{l}\text { Resistance } \\
\text { Current rating } \\
\text { Length of manganin tube between potential leads } \\
\text { Thicketer } \\
\text { Watts pers square centimeter at rated current. } \\
\text { Phase angle at } 60 \text { cycles, computed. }\end{array}$ & $\begin{array}{l}0.0005 \\
1,000 \\
35 \\
8.57 \\
.1 \\
. .27 \\
-.7\end{array}$ & $\begin{array}{rl} & 0.00025 \\
2,500 & 64 \\
15.2 \\
.2 \\
.26 \\
-2.9\end{array}$ & $\begin{array}{l}0.00020 \\
2,500 \\
51 \\
15.2 \\
.2 \\
-2.26 \\
-2.9\end{array}$ \\
\hline
\end{tabular}


Cooling is effected by a stream of oil (about 20 gallons per minute) which is circulated by a centrifugal pump through the annular spaces on each side of the manganin tubes. The linear velocity of the oil is about $65 \mathrm{~cm}$ per second, and the heat transfer is about 0.023 watt per square $\mathrm{cm}$ per degree centigrade temperature rise. Thermocouple measurements have shown that at rated current the manganin operates at a temperature about $11^{\circ} \mathrm{C}$. above that of the oil.

As manganin tubing of the required diameter was not commercially available, the shunts were constructed by bending sheet material to form portions of a circular cylinder of the desired radius. For the smaller tube two pieces each extending the full length of the shunt and subtending an angle of $180^{\circ}$ were used, and for the larger three pieces each covering $120^{\circ}$. The sheets were hard soldered at the ends to narrow copper rings which in turn were soft soldered to the heavy copper tubes which formed the rest of the current circuit. The tubes were annealed at $450^{\circ} \mathrm{C}$. in an atmosphere of nitrogen after all mechanical work on the manganin had been completed.

Because of the unsymmetrical location of the current-terminal slabs there would be a tendency for the current density to be slightly greater in the upper part of both tubes than in the lower. To counteract this effect, a number of holes were drilled through the slabs near the upper side of the tubes (see fig. 14) so as to tend to force the current flow into the other parts of the circumference. A test for the adequacy of this procedure is obtained by sending direct current through the shunt and experimentally plotting some loci of points of equal potential on the surface of the tube near the slabs. These equipotential lines normaliy lie in planes perpendicular to the axis of the tube, and any departure from this condition is an index of nonuniform current distribution. This method also gives a fairly sensitive test of the quality of the soldered joints, a very small crack producing a marked distortion of the equipotential lines.

These shunts are provided with four sets of potential leads spaced $90^{\circ}$ apart around the tubes. Normally the four leads of each equipotential group are connected in parallel to minimize errors from eccentricity and stray fields. As a preliminary check, however, the inductance was measured using each set of leads separately. The values when analyzed by the method given in Section IV (equation (17)) above indicated that the actual current distribution was equivalent to that in a pair of homogeneous tubes having a displacement between their axes of only $0.1 \mathrm{~mm}$ in the case of the smaller and 0.7 $\mathrm{mm}$ in the larger shunt. This is, perhaps, the result of inhomogeneity in the metal rather than of an actual eccentricity of the tubes.

Adjustment of resistance is provided by the method shown in the lower part of Figure 4, using two potential leads connected at points about 2.5 per cent of the resistance apart at one end of each shunt. All potential leads of the group connecting to the same equipotential surface are of the same length and resistance. Those running toward the closed end of the current circuit are of No. 18 manganin wire rolled flat and tied down tightly against the tube. Between the tube and the binding post one group of potential leads is formed by four slender copper or brass tubes, while the corresponding leads of the group from the other end of the shunt are drawn through these tubes, which thus function as conduits and also serve to keep the two leads 


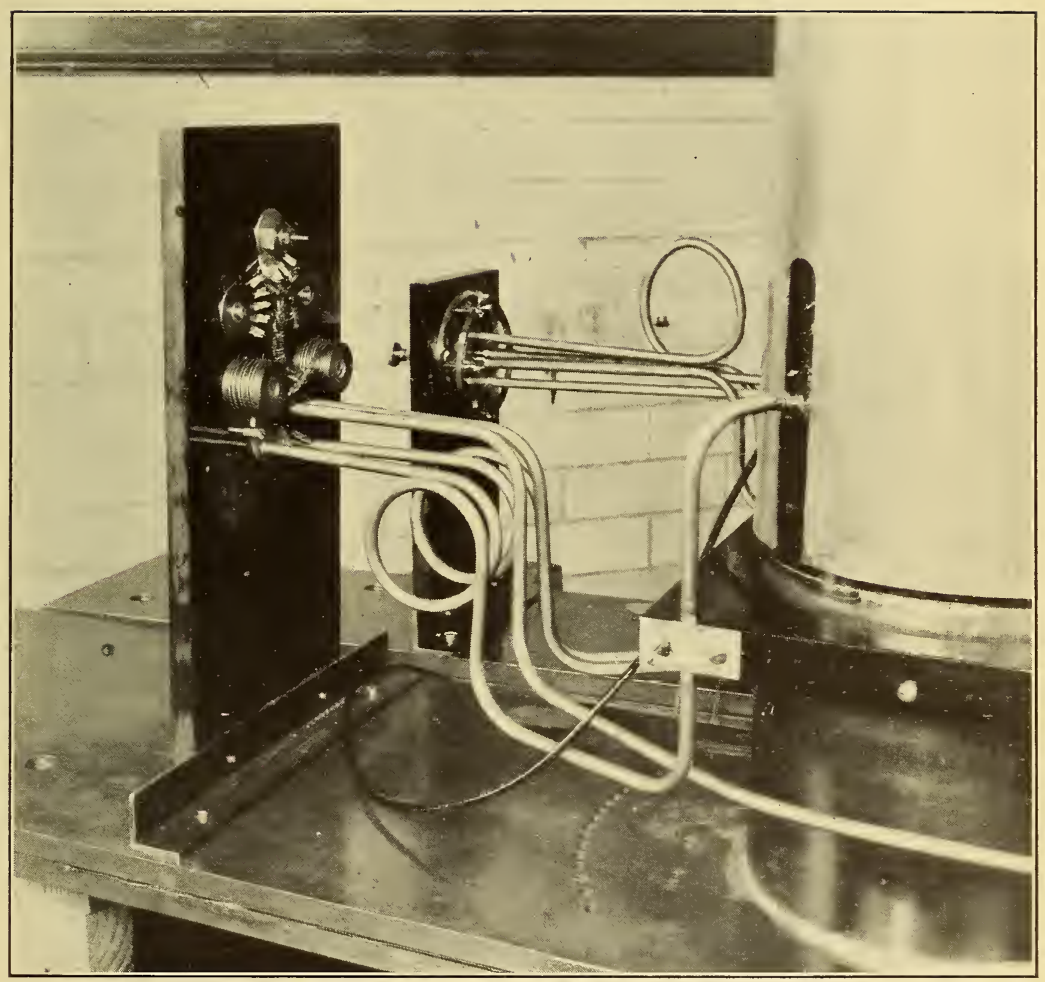

Figure 14.-View of oil-cooled shunts removed from tank and placed with the axis of the current-carrying tubes vertical, to show the under side of the potential terminals, the resistors which serve for the exact adjustment of the resistances, and the tubular potential leads which serve as conduits for those other potential leads which are attached to the shunt along the same element 
of each set close together to avoid any loops which might link with stray fields. For the same reason the binding posts themselves are made concentric.

\section{CONCLUSION}

It will be seen from the foregoing sections that the design of a resistance standard for alternating current need not differ very greatly from that of a resistor for direct current. The prime considerations of resistance and cooling are the same for both. The requirements of a small and definite inductance can usually be met by proper attachment and location of the potential leads without greatly affecting the cooling. To avoid skin-effect errors somewhat more modification in design is required, but the symmetry of construction usually resorted to in alternating-current standards would probably lead to more uniform temperature distribution and better performance than the more conventional direct-current designs.

It is hoped that the descriptions given in Sections VI and VII of the resistors used at this bureau in current-transformer testing may be of assistance to other laboratory workers in preparing satisfactory designs. The choice of the tubular construction for the standards of greatest current-carrying capacity was dictated mainly by the consideration that the inductance would be computable from the dimensions. Now that these resistors are in existence others of different construction but of the same nominal resistance can be compared with them and, hence, there is less necessity for the construction of similar resistors in the future. It may be remarked, however, that the mechanical difficulty of constructing the necessary tubes from sheet material was found to be much less than had been anticipated, and that the advantages of freedom from stray fields and of nearly uniform temperature distribution throughout the working part of the shunt are not to be undervalued.

\section{NOTATION}

$a=$ coordinate of point (or potential lead) from center of rectangle (or strip) measured along shorter axis, or

$a=$ outer radius of inner conductor of concentric main.

$b=$ coordinate of point (or potential lead) from center of rectangle (or strip) measured along longer axis.

$b=$ inner radius of outer conductor of concentric main.

$c=$ outer radius of outer conductor of concentric main.

$D=$ linear dimension.

$D_{13}, D_{14}, D_{x \nu}=$ geometric mean distances between areas 1 and 3,1 and $4, x$ and $y$, etc.

$d=$ thickness of conducting strip.

$d_{12}, d_{13}=$ distances between elements of conductors 1 and 2 , 1 and 3 , etc.

$E=$ electromotive force.

$F_{1}, \mathrm{~F}_{2}, F_{3}, F_{4}=$ functions defined by equations (35), (37), (38), and (39), respectively.

$f=$ frequency.

$g=$ distance between centers of strips (or rectangles). 
$H_{1}, H_{2}=$ heat developed in inner and outer conductors of concentric main.

$I=$ current.

$i=$ current density.

$i_{1}, i_{2}=$ current density in conductors 1,2 .

$i^{\prime}=$ current density in outer conductor of concentric main.

$i_{0}=$ current density at center of inner conductor of con-

$j=\sqrt{-1}$. centric main.

$k=$ constant of equation (12).

$L=$ self inductance.

$L_{1}, L_{2}=$ inductance using potential leads 1,2 .

$L_{l}=$ inductance of shunt of length $l$.

$L_{\infty}=$ inductance of section of length $l$ of a shunt of infinite length.

$l, l_{1}, l_{2}=$ axial lengths .

$M=$ mutual inductance.

$M_{12},{ }_{34}=$ mutual inductance between circuit 12 and circuit 34 .

$m=\sqrt{\frac{8 \pi^{2} f}{\rho}}=\sqrt{\frac{4 \pi \omega}{\rho}}$.

$P=$ power transferred by transformer action between conductors of concentric main.

$p=m \sqrt{\frac{d w}{\pi}}$.

$q=$ displacement of axes.

$R=$ direct-current resistance.

$R^{\prime}=$ alternating current resistance.

$R_{i}=$ alternating-current resistance of inner conductor.

$\Delta R=$ change in resistance between alternating current and direct current.

$r=$ radial distance.

$S_{1}, S_{2}=$ area of conductor 1,2 .

$t=$ constant of equation 12 .

$v=$ volume.

$w=$ width of strip.

$y=$ variable of integration in equation 12 .

$x=g / w$.

$Z=$ impedance.

$\alpha_{1}=$ angle between line joining axes of tubes and line joining axis of outer tube to potential lead 1.

$\mu=$ permeability.

$\rho=$ resistivity.

$\varphi_{1}, \varphi_{2}=$ flux linkages due to current in conductors 1,2 .

$\phi=$ total flux linkages.

$\omega=2 \pi f$.

\section{APPENDIX A.-FORMULAS FOR GEOMETRIC MEAN DISTANCE ${ }^{26}$}

The following list of formulas contains those for most of the combinations of points and narrow rectangles likely to be needed in computing the inductance of shunts formed of manganin strips and

${ }^{26}$ The author is deeply indebted to Prof. F. W. Grover for his painstaking care in verifying these formulas. 
small potential leads. They may be derived as special cases of the more general expression for the geometric mean distance of two symmetrically located rectangles of any thickness given by Rosa ${ }^{27}$ but are more readily arrived at by the direct integration of the equation which defines geometric mean distance.

For the case of strips which have a thickness, $d$, very small compared to their width, $w$, Taylor's theorem may be applied and a power series in $\frac{d^{2}}{w^{2}}$ obtained. The first term of this series is given in each of the equations listed below.

If the geometric mean distances for pairs of lines or points and lines are desired, they can be obtained by merely omitting from the equations given below those terms which contain $\frac{d^{2}}{w^{2}}$ as a factor.

The formulas given here cover only those cases in which the rectangles (or lines) are equal and are placed with their longer axes parallel and with their shorter axes in the same straight line. The following notation is used:

$w=$ length of rectangle (that is, width of strip).

$d=$ width of rectangle (that is, thickness of strip).

$g=$ distance between centers of rectangles.

$a=$ coordinate of point from center of rectangle measured along shorter axis.

$b=$ coordinate of point from center of rectangle measured along longer axis.

$n=\frac{1}{2}-\frac{b}{w}$.

$D=$ geometric mean distance.

\section{Point from narrow rectangle}

(a) General case:

$$
\begin{aligned}
\log _{e} D= & \frac{1}{2}\left(\frac{b}{w}+\frac{1}{2}\right) \log _{e}\left\{a^{2}+\left(b+\frac{w}{2}\right)^{2}\right\} \\
& -\frac{1}{2}\left(\frac{b}{w}-\frac{1}{2}\right) \log _{e}\left\{a^{2}+\left(b-\frac{w}{2}\right)^{2}\right\}-1 \\
& +\frac{a}{w}\left\{\tan ^{-1}\left(\frac{b}{a}+\frac{w}{2 a}\right)-\tan ^{-1}\left(\frac{b}{a}-\frac{w}{2 a}\right)\right\} \\
& -\frac{d^{2}}{24 w}\left\{\frac{\frac{w}{2}-b}{a^{2}+\left(\frac{w}{2}-b\right)^{2}}+\frac{\frac{w}{2}+b}{a^{2}+\left(\frac{w}{2}+b\right)^{2}}\right\}
\end{aligned}
$$

(b) Symmetrical case, point on prolongation of minor axis of rectangle: 


$$
\log _{e} D=\frac{1}{2} \log _{e}\left(a^{2}+\frac{w^{2}}{4}\right)-1+\frac{2 a}{w} \tan ^{-1} \frac{w}{2 a}-\frac{d^{2}}{24\left(a^{2}+\frac{w^{2}}{4}\right)}
$$

(c) Point close to rectangle(but not symmetrical; that is, expansion in $\left.\frac{a}{w}\right) ;$ let $n=\frac{1}{2}-\frac{b}{w}$ :

$$
\begin{aligned}
\log _{e} D & =\log _{e} w+n \log _{e} n+(1-n) \log _{e}(1-n)-1 \\
& +\frac{\pi a}{w}-\frac{a^{2}}{2 n(1-n) w^{2}}-\frac{d^{2}}{24 w^{2} n(1-n)}
\end{aligned}
$$

unless $b= \pm \frac{w}{2}$, in which case

$$
\log _{e} D=\log _{e} w-1+\frac{\pi a}{2 w}-\frac{a^{2}}{2 w^{2}}-\frac{d^{2}}{24\left(w^{2}+a^{2}\right)}
$$

(d) Point very distant from rectangle (that is, expansion in $\frac{w}{a}$ ):

$$
\begin{aligned}
\log _{e} D= & \log _{e} a+\frac{\left\{n^{3}+(1-n)^{3}\right\} w^{2}}{6 a^{2}}-\frac{\left\{n^{5}+(1-n)^{5}\right\} w^{4}}{20 a^{4}} \cdots \\
& -\frac{d^{2}}{24 w^{2}}\left\{\frac{w^{2}}{a^{2}}-\frac{\left(1-3 n+3 n^{2}\right) w^{4}}{a^{4}}\right\}
\end{aligned}
$$

(e) Point near and on prolongation of shorter axis of rectangle (that is, $b=0$, expansion in $\frac{a}{w}$ ):

$$
\log _{e} D=\log _{e} w-1-\log _{e} 2+\frac{\pi a}{w}-\frac{2 a^{2}}{w^{2}}-\frac{d^{2}}{6 w^{2}} \cdots
$$

(f) Point distant from rectangle and on prolongation of shorter axis (that is, $b=0$, expansion in $\frac{w}{a}$ )

$$
\log _{e} D=\log _{e} a+\frac{w^{2}}{24 a^{2}} \cdots-\frac{d^{2}}{24 w^{2}}\left\{\frac{w^{2}}{a^{2}}-\frac{w^{4}}{4 a^{4}} \cdots\right\}
$$

\section{Two rectangles}

(a) General case of equal, symmetrically placed, narrow rectangles (that is, expansion in $\frac{d}{w}$ ):

$$
\begin{aligned}
\log _{e} D= & \frac{1}{2}\left(1-\frac{g^{2}}{w^{2}}\right) \log _{e}\left(g^{2}+w^{2}\right)+\frac{g^{2}}{w^{2}} \log _{e} g-3 / 2 \\
& +\frac{2 g}{w} \tan ^{-1} \frac{w}{g}+\frac{d^{2}}{12 w^{2}} \log _{e} \frac{g^{2}}{g^{2}+w^{2}} \ldots
\end{aligned}
$$


(b) Rectangles close together (that is, expansion in $g / w$ ):

$$
\begin{aligned}
\log _{e} D= & \log _{e} w-\frac{3}{2}+\frac{g^{2}}{w^{2}} \log _{e} \frac{g}{w}+\frac{\pi g}{w}-\frac{3}{2} \frac{g^{2}}{w^{2}} \\
& +\frac{d^{2}}{12 w^{2}}\left\{-\frac{g^{2}}{w^{2}}+\frac{g^{4}}{2 w^{4}} \cdots+2 \log _{e} \frac{g}{w}\right\}
\end{aligned}
$$

(c) Rectangles far apart (that is, expansion in $\frac{w}{g}$ ):

$$
\log _{e} D=\log _{e} g-\frac{w^{2}}{12 g^{2}}+\frac{w^{4}}{60 g^{4}}+\frac{d^{2}}{12 w^{2}}\left\{-\frac{w^{2}}{g^{2}}+\frac{w^{4}}{2 g^{4}} \ldots\right\}
$$

(d) Rectangles coinciding (that is, g. m. d. of rectangle from itself):

1. For any rectangle:

$$
\begin{aligned}
\log _{e} D= & \log _{e} \sqrt{w^{2}+d^{2}}-\frac{1}{6} \frac{d^{2}}{w^{2}} \log _{e} \sqrt{1+\frac{w^{2}}{d^{2}}}-\frac{1}{6} \frac{w^{2}}{d^{2}} \log _{e} \sqrt{1+\frac{d^{2}}{w^{2}}} \\
& +\frac{2}{3} \frac{d}{w} \tan ^{-1} \frac{w}{d}+\frac{2}{3} \frac{w}{d} \tan ^{-1} \frac{d}{w}-\frac{25}{12}
\end{aligned}
$$

2. For narrow rectangle (that is, expansion in $\left.\frac{d}{w}\right)$ :

$$
\log _{e} D=\log _{e} w-\frac{3}{2}+\frac{\pi d}{3 w}+\frac{d^{2}}{6 w^{2}} \log _{e} \frac{d}{w}-\frac{25 d^{2}}{72 w^{2}} \cdots
$$

3. For line of length $w$ (that is, $d=0$ ):

Washington, July 19, 1929.

$$
\log _{e} D=\log _{e} w-\frac{3}{2}
$$

